\title{
Resonant absorption of the slow sausage wave in the slow continuum
}

\author{
D. J. Yu, T. Van Doorsselaere, and M. Goossens
}

\begin{abstract}
Centre for mathematical Plasma Astrophysics, Department of Mathematics, KU Leuven, Celestijnenlaan 200B bus 2400, 3001 Leuven, Belgium e-mail: [daejung.yu; tom.vandoorsselaere;marcel.goossens]@kuleuven.be
\end{abstract}

Received 25 December 2016 / Accepted 6 March 2017

\begin{abstract}
Aims. General analytical formulas for the damping rate by resonant absorption of slow sausage modes in the slow (cusp) continuum are derived and the resonant damping of the slow surface mode under photospheric conditions is investigated.

Methods. The connection formula across the resonant layer is used to derive the damping rate for the slow sausage mode in the slow continuum by assuming a thin boundary.

Results. It is shown that the effect of the resonant damping on the slow surface sausage mode in the slow continuum, which has been underestimated in previous interpretations, could be efficient under magnetic pore conditions. A simplified analytical formula for the damping rate of slow surface mode in the long wavelength limit is derived. This formula can be useful for a rough estimation of the damping rate due to resonant absorption for observational wave damping.
\end{abstract}

Key words. magnetohydrodynamics (MHD) - Sun: photosphere - Sun: oscillations

\section{Introduction}

It is well-known that magnetohydrodynamic (MHD) waves are omnipresent in the lower solar atmosphere. Hence clarifying how much wave energy transfers to and dissipates in the upper atmosphere is an important issue (e.g. Kanoh et al. 2016). Because the waves in the lower atmosphere are related to the motions of sunspots, magnetic pores, and granules, slow waves are of particular interest. Recently, the advances in instrumental technologies have made it possible to observe slow waves and their damping. After the first identification of sausage wave in magnetic pores (Dorotovič et al. 2008), studies on the characteristics of sausage modes in different situations followed (e.g. Morton et al. 2011; Dorotovič et al. 2014; Moreels et al. 2015a). The identification of the wave mode is based on simplified modelling of the magnetic flux structure into a long, straight, cylindrical flux tube. This theoretical approach allows to specify, for example, the dispersion curves, phase relations between different physical parameters for each wave modes, and wave energy flux (Edwin \& Roberts 1983; Evans \& Roberts 1990; Fujimura \& Tsuneta 2009; Moreels \& Van Doorsselaere 2013; Moreels et al. 2013, 2015a,b; Freij et al. 2016).

Some observations show rapid damping of the slow waves (e.g. Krishna Prasad et al. 2014; Grant et al. 2015). The wave damping is thought to be due to thermal conduction, compressional viscosity, optically thin radiation, and area (field line) divergence, while the gravitational stratification causes an increase of the wave amplitude. De Moortel \& Hood (2003) compared the effect of thermal conduction with that of compressive viscosity under coronal conditions and found that thermal conduction, when it is dominant, causes minimum damping time (or length). They subsequently investigated the effect of optically thin radiation, gravitational stratification, and area divergence on the wave damping (De Moortel \& Hood 2004). It was shown that the gravitational stratification has a crucial role in increasing the damping length and a general area divergence can also lead to a significant damping of the wave amplitude. Interestingly, frequency dependence of the wave damping was recently found by Krishna Prasad et al. (2014), which was interpreted as a sign of damping by thermal conduction (Mandal et al. 2016). Independently to these mechanisms, to explain the strong damping reported in Grant et al. (2015), decay as a leaky mode, high reflection at the cut-off, and mode conversion into fast mode at the transition region are suggested.

On the other hand, resonant absorption can also cause rapid decay of waves. This mechanism is considered as a strong candidate for explaining the damped transverse (kink) oscillations of the coronal loops (e.g. Goossens et al. 2002, 2008). In addition to the investigation of the damping rate (see e.g. Van Doorsselaere et al. 2004; Soler et al. 2013), it is also important to know how much wave energy can be absorbed in the resonant layer (see e.g. Terradas et al. 2006; Arregui et al. 2011; Soler et al. 2013; Yu \& Van Doorsselaere 2016). In the lower atmosphere of the Sun, the presence of the slow resonance along with or without the Alfvén resonance can crucially affect resonant absorption (e.g. Keppens 1995, 1996). Cadez et al. (1997) studied resonant absorption of slow and fast magnetosonic waves in the presence of local Alfvén or slow continua in Cartesian geometry, while Ruderman (2009) focused on the wave motion at the resonance layer in the presence of both the slow and Alfvén resonances when they are very close to one another. The competition of resonant absorption with viscosity and thermal conductivity was dealt with by Ruderman et al. (2000) and it was shown that the resonant absorption is dominant over viscosity damping and damping by thermal conductivity when $l \zeta R_{\mathrm{e}} \gg 1$ where $l$ is the thickness of the transitional layer, $\zeta$ the wavenumber, and $R_{\mathrm{e}}$ the parallel total Reynolds number. Soler et al. (2009) 
considered the oscillation of a kink mode in a filament thread, which undergoes resonant damping in both the Alfvén and slow continua in cylindrical geometry. They found that the damping due to the slow resonance is negligibly weak compared to that due to the Alfvén resonance. Very recently, the study on resonant absorption of axisymmetric modes $(m=0)$ in the Alfvén continuum in the presence of a twisted magnetic field showed that both the longitudinal magnetic field and the density have crucial roles in the wave damping (Giagkiozis et al. 2016). If there is no azimuthal magnetic field, only resonant absorption in the slow (cusp) resonance for axisymmetric modes is possible.

As shown in the paper by Soler et al. (2009), it is often believed that the effect of slow resonance on the wave damping is much lower than that of Alfvén resonance, but for the mode $m=0$, the characteristics of the wave damping due to resonant absorption is not well known. Therefore we investigate this issue theoretically, especially for the slow surface mode.

The paper is structured as follows. In Sect. 2, we obtain the dispersion relation for the magnetic pore conditions by assuming that the plasma is homogeneous inside and outside the pore. In Sect. 3, we derive the damping rate for the slow surface wave by considering a thin transitional layer between inner and outer regions of the pore by using the connection formula. In this section, we also introduce the model configuration for the transitional layer. The results are shown in Sect. 4. We conclude the paper in Sect. 5.

\section{Dispersion relation}

We consider a uniform axisymmetric cylinder of magnetic field $B_{\mathrm{i}} \hat{z}$ inside and $B_{\mathrm{e}} \hat{z}$ outside the flux tube in the absence of steady flow. The inner (i) and outer (e) regions of the flux tube satisfy the pressure balance equation

$p_{\mathrm{i}}+\frac{B_{\mathrm{i}}^{2}}{2 \mu_{0}}=p_{\mathrm{e}}+\frac{B_{\mathrm{e}}^{2}}{2 \mu_{0}}$,

where $\mu_{0}$ is the magnetic permeability and $p$ is the plasma pressure. The density contrast between the inside and the outside of the flux tube is given as

$\chi=\frac{\rho_{\mathrm{e}}}{\rho_{\mathrm{i}}}=\frac{2 v_{\mathrm{si}}^{2}+\gamma v_{\mathrm{Ai}}^{2}}{2 v_{\mathrm{se}}^{2}+\gamma v_{\mathrm{Ae}}^{2}}$,

where $v_{\mathrm{s}}=\sqrt{\gamma p / \rho}$ is the sound speed, $v_{\mathrm{A}}=B / \sqrt{\mu_{0} \rho}$ the Alfvén speed, $\gamma$ the ratio of the specific heats (adiabatic index), and $\rho$ the density.

We begin with linearised ideal MHD with the dependence $\exp \left(k_{z} z+m \phi-\omega t\right)$ where $k_{z}$ is the longitudinal wavenumber, $m$ the azimuthal wavenumber, and $\omega$ the angular frequency of the wave. Let us first deal with the case of a true discontinuity at the boundary $r=R$ where the inner region $(r<R)$ and the outer region $(r>R)$ are homogeneous. At the boundary, the density $\rho$ changes abruptly from its internal value $\rho_{\text {i }}$ to its external value $\rho_{\mathrm{e}}$. The dispersion relation is then obtained by imposing continuity of total pressure $P$ and of the radial component of the Lagrangian displacement $\xi_{r}$ (Sakurai et al. 1991; Goossens et al. 1992):

$[P]=P_{\mathrm{e}}-P_{\mathrm{i}}=0$,

$\left[\xi_{r}\right]=\frac{1}{\rho_{\mathrm{e}}\left(\omega^{2}-\omega_{\mathrm{Ae}}^{2}\right)} \frac{\mathrm{d} P_{\mathrm{e}}}{\mathrm{d} r}-\frac{1}{\rho_{\mathrm{i}}\left(\omega^{2}-\omega_{\mathrm{Ai}}^{2}\right)} \frac{\mathrm{d} P_{\mathrm{i}}}{\mathrm{d} r}=0$, where $\omega_{\mathrm{A}}=k_{z} v_{\mathrm{A}}$ is the local Alfvén frequency and we have used the relation (see e.g. Sakurai et al. 1991; Goossens et al. 1992)

$\xi_{r}=\frac{1}{\rho\left(\omega^{2}-\omega_{\mathrm{A}}^{2}\right)} \frac{\mathrm{d} P}{\mathrm{~d} r}$.

For the surface wave eigenmodes, Eq. (4) can be reduced to

$\frac{A_{\mathrm{e}} k_{\mathrm{e}} K_{m}^{\prime}\left(k_{\mathrm{e}} R\right)}{\rho_{\mathrm{e}}\left(\omega^{2}-\omega_{\mathrm{Ae}}^{2}\right)}-\frac{A_{\mathrm{i}} k_{\mathrm{i}} I_{m}^{\prime}\left(k_{\mathrm{i}} R\right)}{\rho_{\mathrm{i}}\left(\omega^{2}-\omega_{\mathrm{Ai}}^{2}\right)}=0$,

where the prime denotes the derivative with respect to the entire argument, $I_{m}$ and $K_{m}$ are modified Bessel functions of first and second kinds, respectively, and $A_{\mathrm{i}, \mathrm{e}}$ is the matching coefficient. The radial wave numbers, $k_{\mathrm{i}}$ and $k_{\mathrm{e}}$, are given by

$k_{\mathrm{i}}^{2}=-\frac{\left(\omega^{2}-\omega_{\mathrm{si}}^{2}\right)\left(\omega^{2}-\omega_{\mathrm{Ai}}^{2}\right)}{\left(v_{\mathrm{si}}^{2}+v_{\mathrm{Ai}}^{2}\right)\left(\omega^{2}-\omega_{\mathrm{Ci}}^{2}\right)}, \quad k_{\mathrm{e}}^{2}=-\frac{\left(\omega^{2}-\omega_{\mathrm{se}}^{2}\right)\left(\omega^{2}-\omega_{\mathrm{Ae}}^{2}\right)}{\left(v_{\mathrm{se}}^{2}+v_{\mathrm{Ae}}^{2}\right)\left(\omega^{2}-\omega_{\mathrm{Ce}}^{2}\right)}$,

where $\omega_{\mathrm{C}}=k_{z} v_{\mathrm{C}}$, the cusp frequency, $v_{\mathrm{C}}=\sqrt{v_{\mathrm{A}}^{2} v_{\mathrm{s}}^{2} /\left(v_{\mathrm{A}}^{2}+v_{\mathrm{S}}^{2}\right)}$, the cusp speed, and $\omega_{\mathrm{s}}=k_{z} v_{\mathrm{s}}$.

From the continuity of total pressure $\left(A_{\mathrm{e}} K_{m}=A_{\mathrm{i}} I_{m}\right)$, we obtain the dispersion relation $D_{m}=0$ for azimuthal wave number $m$ :

$D_{m}=\rho_{\mathrm{i}}\left(\omega^{2}-\omega_{\mathrm{Ai}}^{2}\right)-\rho_{\mathrm{e}}\left(\omega^{2}-\omega_{\mathrm{Ae}}^{2}\right)\left(\frac{k_{\mathrm{i}}}{k_{\mathrm{e}}}\right) Q_{m}=0$,

where

$Q_{m}=\frac{I_{m}^{\prime}\left(k_{\mathrm{i}} R\right) K_{m}\left(k_{\mathrm{e}} R\right)}{I_{m}\left(k_{\mathrm{i}} R\right) K_{m}^{\prime}\left(k_{\mathrm{e}} R\right)}$.

We are concerned with the sausage mode $(m=0)$ in the present paper. From Eq. (8) we find that the eigenfrequency satisfies the following relation

$\omega^{2}=\frac{\rho_{\mathrm{i}} \omega_{\mathrm{Ai}}^{2}-\rho_{\mathrm{e}} \omega_{\mathrm{Ae}}^{2}\left(\frac{k_{\mathrm{i}}}{k_{\mathrm{e}}}\right) Q_{0}}{\rho_{\mathrm{i}}-\rho_{\mathrm{e}}\left(\frac{k_{\mathrm{i}}}{k_{\mathrm{e}}}\right) Q_{0}}$.

This is a complicated equation for $\omega$ since $\omega$ appears in $k_{\mathrm{i}}, k_{\mathrm{e}}$, and $Q_{0}$. We need to solve it numerically as has been done by, for example, Edwin \& Roberts (1983). For the body modes, we need to use Bessel function $J_{m}$ instead of $I_{m}$ and + sign instead of - sign in $k_{i}^{2}$ in Eq. (7).

We use the values of parameters for the inner magnetic pore described in Grant et al. (2015) and typical values of parameters for the outer region of the magnetic pore, which were not present in the paper. We plot several eigenmodes for $m=0$ in Fig. 1: fast surface mode (fs), eight slow body modes among multiple solutions (sb1-sb4), and slow surface mode (ss). It follows from the figure that the slow surface mode is in the cusp frequency range $0=v_{\mathrm{Ce}}<v_{\mathrm{ss}} \leq v_{\mathrm{Ci}}$ while the slow body modes are in the range $v_{\mathrm{Ci}}<v_{\mathrm{sb}} \leq v_{\mathrm{si}}$. This means that when the discontinuity is replaced by continuous variation, the slow surface wave (ss) has its frequency in the slow (cup) continuum and hence will be resonantly damped.

In the incompressible limit $\left(v_{\mathrm{si}}, v_{\mathrm{se}} \rightarrow \infty\right), k_{\mathrm{e}}\left(k_{\mathrm{i}}\right) \rightarrow k_{z}$, then Eq. (10) reduces to (see e.g. Edwin \& Roberts 1983)

$\omega^{2}=\frac{\rho_{\mathrm{i}} \omega_{\mathrm{Ai}}^{2}-\rho_{\mathrm{e}} \omega_{\mathrm{Ae}}^{2} Q_{0}}{\rho_{\mathrm{i}}-\rho_{\mathrm{e}} Q_{0}}$. 
D. J. Yu et al.: Resonant absorption of the slow sausage wave in the slow continuum

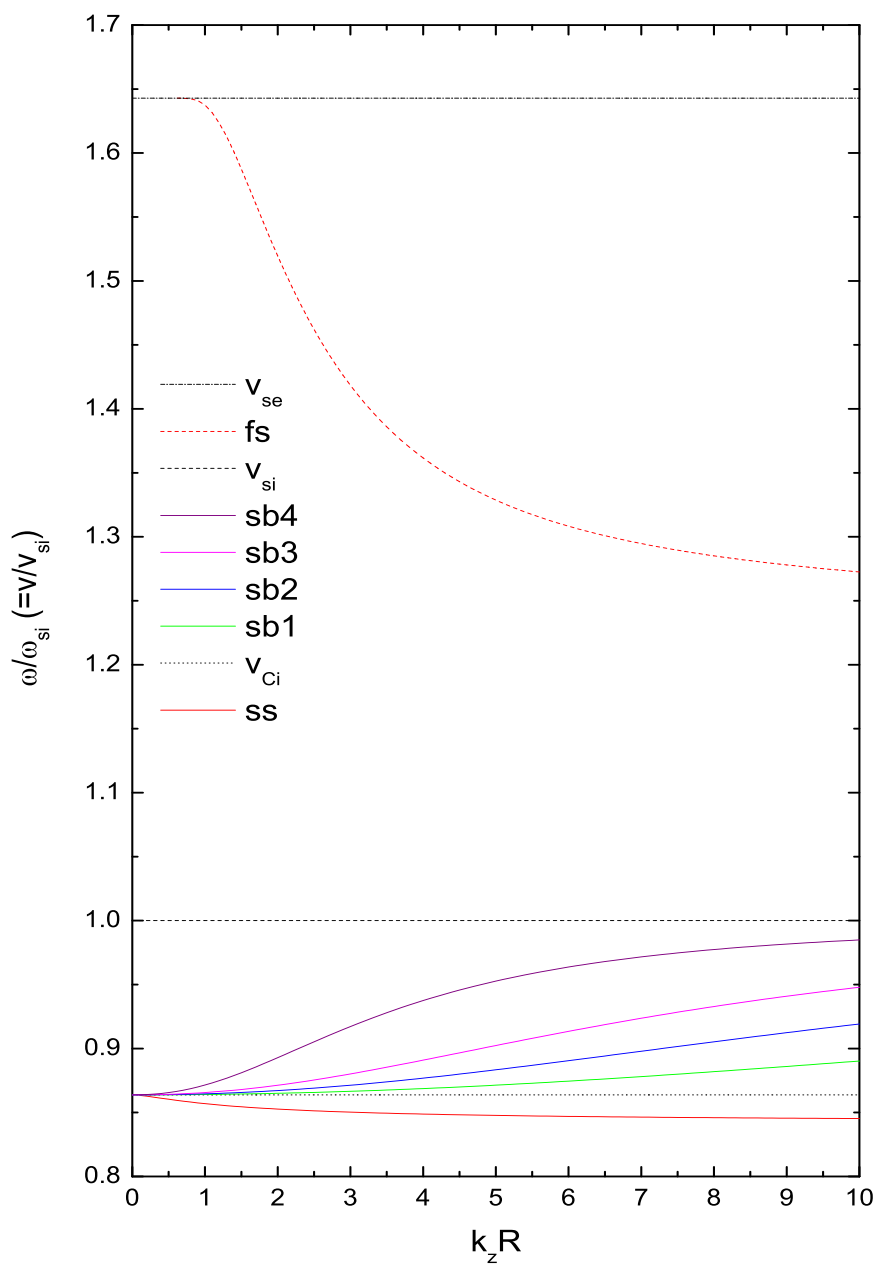

Fig. 1. Phase speed $\omega / \omega_{\mathrm{si}}$ as a function of $k_{z} R$ for a fast surface mode (fs), 8 slow body modes (sb1-sb4), and a slow surface mode (ss) under magnetic pore conditions when $v_{\mathrm{Ae}}=0 \mathrm{~km} \mathrm{~s}^{-1}, v_{\mathrm{Ai}}=$ $12 \mathrm{~km} \mathrm{~s}^{-1}, v_{\mathrm{se}}=11.5 \mathrm{~km} \mathrm{~s}^{-1}, v_{\mathrm{si}}=7 \mathrm{~km} \mathrm{~s}^{-1}, v_{\mathrm{Ce}}=0 \mathrm{~km} \mathrm{~s}^{-1}$, $v_{\mathrm{Ci}}=6.0464 \mathrm{~km} \mathrm{~s}^{-1}\left(=0.86378 v_{\mathrm{si}}\right), \beta_{\mathrm{i}}=(2 / \gamma)\left(v_{\mathrm{si}} / v_{\mathrm{Ai}}\right)^{2}=0.4083$ and $\beta_{\mathrm{e}}=(2 / \gamma)\left(v_{\mathrm{se}} / v_{\mathrm{Ae}}\right)^{2}=\infty$. All quantities are normalized by $v_{\mathrm{si}}$.

In this limit, the right hand side of Eq. (11) has no dependence on $\omega$.

For $k_{z} R<1$, from Eq. (A.5), Eq. (11) reduces

$\omega^{2}=\frac{\rho_{\mathrm{i}} \omega_{\mathrm{Ai}}^{2}-\rho_{\mathrm{e}} \omega_{\mathrm{Ae}}^{2} \frac{k_{z}^{2} R^{2}}{2} \ln \left(k_{z} R\right)}{\rho_{\mathrm{i}}-\rho_{\mathrm{e}} \frac{k_{z}^{2} R^{2}}{2} \ln \left(k_{z} R\right)}$,

where we have used $\ln \left(k_{z} R / 2\right)+\gamma_{\mathrm{e}} \approx \ln \left(k_{z} R\right)$.

For $k_{z} R \ll 1$ and $\omega \approx \omega_{\mathrm{Ci}}$ we can assume $\omega^{2}=\omega_{\mathrm{Ci}}^{2}+\alpha$, then the condition $D_{0}=0$ (Eq. (10)) leads with the aid of Eq. (A.5) (dropping all higher order terms of $k_{\mathrm{i}} R$ and $k_{\mathrm{e}} R$ ) to

$\rho_{\mathrm{i}}\left(\omega_{\mathrm{Ci}}^{2}-\omega_{\mathrm{Ai}}^{2}\right)-\rho_{\mathrm{e}}\left(\omega_{\mathrm{Ci}}^{2}-\omega_{\mathrm{Ae}}^{2}\right) \frac{k_{\mathrm{i}}^{2} R^{2}}{2} \ln \left(k_{\mathrm{e}} R\right)=0$.

Equation (13) can further be reduced to

$$
\begin{aligned}
\left(\omega_{\mathrm{Ci}}^{2}-\omega_{\mathrm{Ai}}^{2}\right) \alpha & +\frac{\chi k_{z}^{2} R^{2}}{2} \frac{\left(\omega_{\mathrm{Ci}}^{2}-\omega_{\mathrm{si}}^{2}\right)\left(\omega_{\mathrm{Ci}}^{2}-\omega_{\mathrm{Ae}}^{2}\right)\left(\omega_{\mathrm{Ci}}^{2}-\omega_{\mathrm{Ai}}^{2}\right)}{\left(\omega_{\mathrm{si}}^{2}+\omega_{\mathrm{Ai}}^{2}\right)} \\
& \times \ln \left(k_{z} R\right)=0,
\end{aligned}
$$

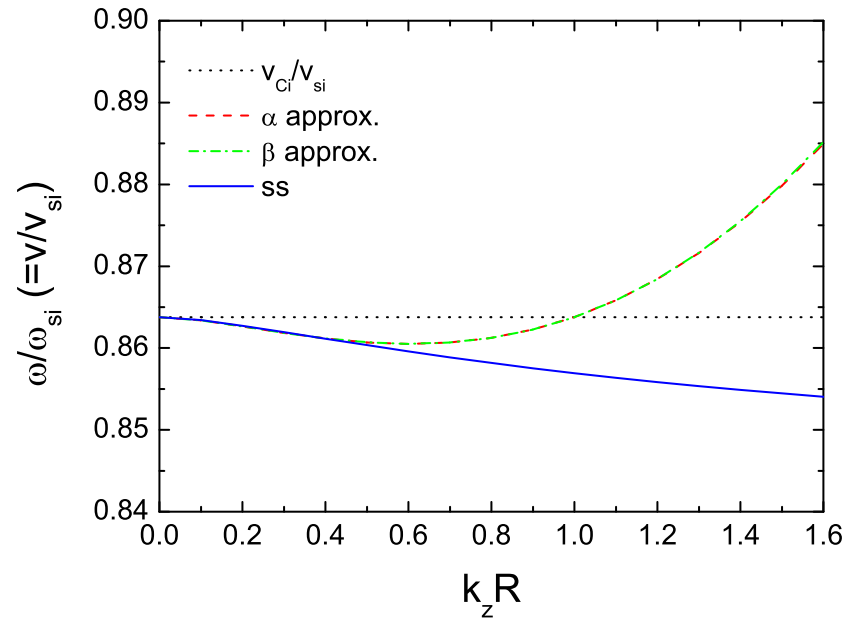

Fig. 2. Phase speed $\omega / \omega_{\mathrm{si}}$ for the slow surface mode (ss) versus $k_{z} R$. We compare the numerical results (Eq. (10)) with those by using the analytical formulas $\alpha$ (Eq. (17)) and $\beta$ (Eq. (18)). The $\alpha$ and $\beta$ are indistinguishable in the figure. The parameters are the same as in the previous figure.

by applying

$k_{\mathrm{i}}^{2} \approx-k_{z}^{2} \frac{\left(\omega_{\mathrm{Ci}}^{2}-\omega_{\mathrm{Si}}^{2}\right)\left(\omega_{\mathrm{Ci}}^{2}-\omega_{\mathrm{Ai}}^{2}\right)}{\alpha\left(\omega_{\mathrm{si}}^{2}+\omega_{\mathrm{Ai}}^{2}\right)}$,
$k_{\mathrm{e}} \approx k_{z} \sqrt{-\frac{\left(\omega_{\mathrm{Ci}}^{2}-\omega_{\mathrm{se}}^{2}\right)\left(\omega_{\mathrm{Ci}}^{2}-\omega_{\mathrm{Ae}}^{2}\right)}{\left(\omega_{\mathrm{se}}^{2}+\omega_{\mathrm{Ae}}^{2}\right)\left(\omega_{\mathrm{Ci}}^{2}-\omega_{\mathrm{Ce}}^{2}\right)}}=k_{z} n_{z}$,

where $\chi=\rho_{\mathrm{e}} / \rho_{\mathrm{i}}$. Here $\alpha$ appears only in $k_{\mathrm{i}}^{2}$, which leads to the simple expression, Eq. (14).

We then obtain for $\alpha$

$$
\begin{aligned}
\alpha & =-\frac{\chi}{2} \frac{\left(\omega_{\mathrm{Ci}}^{2}-\omega_{\mathrm{si}}^{2}\right)\left(\omega_{\mathrm{Ci}}^{2}-\omega_{\mathrm{Ae}}^{2}\right)}{\left(\omega_{\mathrm{si}}^{2}+\omega_{\mathrm{Ai}}^{2}\right)} k_{z}^{2} R^{2} \ln \left(k_{z} R\right) \\
& =\frac{\chi}{2} \frac{\omega_{\mathrm{Ci}}^{4}}{\omega_{\mathrm{Ai}}^{4}}\left(\omega_{\mathrm{Ci}}^{2}-\omega_{\mathrm{Ae}}^{2}\right) k_{z}^{2} R^{2} \ln \left(k_{z} R\right),
\end{aligned}
$$

which automatically yields the formula for $k_{\mathrm{i}}^{2}$ :

$k_{\mathrm{i}}^{2}=\frac{2\left(\omega_{\mathrm{Ci}}^{2}-\omega_{\mathrm{Ai}}^{2}\right)}{\chi\left(\omega_{\mathrm{Ci}}^{2}-\omega_{\mathrm{Ae}}^{2}\right) R^{2} \ln \left(k_{z} R\right)}=\frac{-2 \omega_{\mathrm{Ci}}^{2} \omega_{\mathrm{Ai}}^{2}}{\chi \omega_{\mathrm{si}}^{2}\left(\omega_{\mathrm{Ci}}^{2}-\omega_{\mathrm{Ae}}^{2}\right) R^{2} \ln \left(k_{z} R\right)}$.

When we alternatively use $\omega=\omega_{\mathrm{Ci}}+\beta$, we get for $\beta$ (see Moreels et al. 2015a)

$\beta=\frac{\chi}{4} \frac{\omega_{\mathrm{Ci}}^{3}}{\omega_{\mathrm{Ai}}^{4}}\left(\omega_{\mathrm{Ci}}^{2}-\omega_{\mathrm{Ae}}^{2}\right) k_{z}^{2} R^{2} \ln \left(k_{z} R\right)$,

where we have used the relation $\omega^{2}=\omega_{\mathrm{Ci}}^{2}+\alpha=\left(\omega_{\mathrm{Ci}}+\beta\right)^{2} \approx$ $\omega_{\mathrm{Ci}}^{2}+2 \beta \omega_{\mathrm{Ci}}$. These two approximations are identical within first order. In Fig. 2, we compare the $\alpha$ approximation (Eq. (17)) with the numerical result and the $\beta$ approximation (Eq. (19)) for the frequency of the slow surface (ss) mode (Eq. (10)). The two approximations give almost the same values for the range of small $k_{z} R$, indistinguishable in the figure. The difference between the numerical result and the two approximations are less than $0.8 \%$ for $k_{z} R \leq 1$. Although it still gives small error for $k_{z} R>1$, the approximations are not reasonable anymore when $k_{z} R>1$ because the phase speed crosses over the line $v_{\mathrm{Ci}}$, entering into the body mode regime. 


\section{Resonant absorption in the slow continuum}

In the previous section, we consider a discontinuous model with two uniform regions. However in reality there exists an inhomogeneous layer between them, which may affect the wave dynamics as we show in this section. One way to deal with this problem is to derive and then solve the differential equations for the relevant quantities by starting from the ideal MHD equations. For $m=0$ with a longitudinal straight magnetic field and with the dependence $\exp \left(k_{z} z-\omega t\right)$ as considered in previous section, we can derive a second-order ordinary differential equation for $\xi_{r}$ (for a more detailed derivation see e.g. Sakurai et al. 1991; Goossens et al. 1992; Giagkiozis et al. 2016):

$\frac{\mathrm{d}}{\mathrm{d} r}\left[\frac{D}{r C} \frac{\mathrm{d}\left(r \xi_{r}\right)}{\mathrm{d} r}\right]+\rho\left(\omega^{2}-\omega_{\mathrm{A}}^{2}\right) \xi_{r}=0$,

where

$D=\rho\left(\omega^{2}-\omega_{\mathrm{A}}^{2}\right)\left(\omega^{2}-\omega_{\mathrm{C}}^{2}\right)\left(v_{\mathrm{s}}^{2}+v_{\mathrm{A}}^{2}\right)$,

$C=\omega^{4}-k_{z}^{2}\left(v_{\mathrm{s}}^{2}+v_{\mathrm{A}}^{2}\right)\left(\omega^{2}-\omega_{\mathrm{C}}^{2}\right)$.

This differential equation has a singularity at $\omega=\omega_{\mathrm{C}}(r)$ where resonant absorption can occur. Due to the presence of the transitional layer, the value of $\omega_{\mathrm{C}}\left(v_{\mathrm{C}}\right)$ changes continuously from $\omega_{\mathrm{Ci}}\left(v_{\mathrm{Ci}}\right)$ to $\omega_{\mathrm{Ce}}\left(v_{\mathrm{Ce}}\right)$. This regime is called the slow (cusp) continuum. For the photospheric conditions such as the magnetic pore conditions, we have the relationship $v_{\mathrm{Ce}}<v_{\mathrm{Ae}}<v_{\mathrm{Ci}}<$ $v_{\mathrm{si}}<v_{\mathrm{se}}<v_{\mathrm{Ai}}$ (see Fig. 1) and there exist no modes for $v<v_{\mathrm{Ae}}$ and $v>v_{\mathrm{se}}$. For the slow surface mode $v_{\mathrm{Ce}}<v_{\mathrm{ss}}<v_{\mathrm{Ci}}$, so the slow surface mode may undergo resonant absorption in the slow continuum.

The situation totally changes when we consider coronal configurations, $v_{\mathrm{Ce}}<v_{\mathrm{se}}<v_{\mathrm{Ci}}<v_{\mathrm{si}}<v_{\mathrm{Ai}}<v_{\mathrm{Ae}}$. Coronal conditions do not allow wave modes for $v<v_{\mathrm{Ci}}, v_{\mathrm{si}}<v<v_{\mathrm{Ai}}$, and $v>v_{\mathrm{Ae}}$. Therefore the slow surface mode $\left(v<v_{\mathrm{Ci}}\right)$ is not allowed to exist in the corona (see e.g. Figs. 3 and 4 in Edwin \& Roberts 1983) and, as a result, no resonant absorption for the slow surface mode is possible there. There exists a cut-off region for the slow surface wave between the photosphere and the corona, where the slow surface mode would have total reflection. On the other hand, the body modes can exist in both atmospheres when $v_{\mathrm{Ci}}<v_{\mathrm{sb}}<v_{\mathrm{si}}$, so the slow body modes can propagate into the corona from the photosphere.

In general, we need to solve Eq. (20) to obtain the required information. However, when the transitional layer is thin compared to the radius of the tube and our interest is on the strength of the wave damping, we can apply an analytical method, which is introduced in the next subsection.

\subsection{Connection formula}

In Sect. 2, we point out that the eigenfrequency $\omega_{r}\left(v_{\mathrm{ss}}\right)$ of the slow surface mode is smaller than $\omega_{\mathrm{Ci}}\left(v_{\mathrm{Ci}}\right)$, so that this mode will undergo resonant damping when there is a non-uniform layer at the boundary. When there is resonant absorption (damping) the dispersion relation has an additional imaginary part. Sakurai et al. (1991) and Goossens et al. (1992) had developed the analytical formula to connect the damping effect due to the resonant absorption to the dispersion relation.

We replace the discontinuity of density at $r=R$ with a continuous variation in a non-uniform layer $[R-l / 2, R+l / 2]$ from $\rho_{\mathrm{i}}$ to $\rho_{\mathrm{e}}$. The thickness of the non-uniform layer is $l$. A fully nonuniform flux tube corresponds to $l=2 R$. Here we use the thin boundary approximation so that the analytic solutions for $P$ and $\xi_{r}$ can be used in the intervals $[0, R-l / 2]$ and $[R+l / 2, \infty[$. In this way we avoid numerical integration of the ideal MHD equations. The connection formula for the slow continuum is given by

$[P]=0$,

$\left[\xi_{r}\right]=-\mathrm{i} \pi \frac{k_{z}^{2}}{\rho_{c}\left|\triangle_{c}\right|}\left(\frac{v_{\mathrm{s} c}^{2}}{v_{\mathrm{s} c}^{2}+v_{\mathrm{A} c}^{2}}\right)^{2} P_{c}$,

where subscript $c$ denotes the position of slow resonance $\left(r=r_{c}\right)$ and $\triangle_{c}=d\left(\omega^{2}-\omega_{\mathrm{C}}^{2}\right) /\left.\mathrm{d} r\right|_{r=r_{c}}$. The condition for $P$ is the same as before (Eq. (3)).

Since we use the thin boundary approximation $(l \ll R)$, $r_{c}$ is approximately equal to $R$ which we use in the calculation. Of course, when we consider the thick boundary, $r_{c}$ can differ from $R$. From Eqs. (4) and (24) we obtain

$\frac{P_{\mathrm{e}}^{\prime}}{\rho_{\mathrm{e}}\left(\omega^{2}-\omega_{\mathrm{Ae}}^{2}\right)}-\frac{P_{\mathrm{i}}^{\prime}}{\rho_{\mathrm{i}}\left(\omega^{2}-\omega_{\mathrm{Ai}}^{2}\right)}=-\mathrm{i} \pi \frac{k_{z}^{2}}{\rho_{c}\left|\Delta_{c}\right|}\left(\frac{v_{\mathrm{s} c}^{2}}{v_{\mathrm{s} c}^{2}+v_{\mathrm{A} c}^{2}}\right)^{2} P_{c}$.

\subsection{Analytical solution for the damping rate of the slow surface mode}

Here we develop analytical formulae for the damping rate of the slow mode. For the surface mode which is of interest, Eq. (25) can be reduced to

$$
\begin{aligned}
\frac{A_{\mathrm{e}} k_{\mathrm{e}} K_{m}^{\prime}\left(k_{\mathrm{e}} R\right)}{\rho_{\mathrm{e}}\left(\omega^{2}-\omega_{\mathrm{Ae}}^{2}\right)} & -\frac{A_{\mathrm{i}} k_{\mathrm{i}} I_{m}^{\prime}\left(k_{\mathrm{i}} R\right)}{\rho_{\mathrm{i}}\left(\omega^{2}-\omega_{\mathrm{Ai}}^{2}\right)} \\
& +\frac{\mathrm{i} \pi k_{z}^{2}}{\rho_{c} \mid \triangle_{c}}\left(\frac{v_{\mathrm{s} c}^{2}}{v_{\mathrm{s} c}^{2}+v_{\mathrm{A} c}^{2}}\right)^{2} A_{\mathrm{e}} K_{m}\left(k_{\mathrm{e}} R\right)=0,
\end{aligned}
$$

where we have used the continuity of $P\left(P_{\mathrm{i}}=P_{\mathrm{e}}=P_{c}\right)$ and $A_{\mathrm{i}, \mathrm{e}, \mathrm{c}}$ is the matching coefficient.

As before for the discontinuous case we can eliminate the coefficients $A_{\mathrm{i}}, A_{\mathrm{e}}$ to arrive at the dispersion relation. The dispersion function $D_{m}$ has a real and an imaginary part. Eliminating the matching coefficients by using the continuity of the total pressure, we have the dispersion relation $D_{m}=0$ which is

$$
\begin{aligned}
& \rho_{\mathrm{i}}\left(\omega^{2}-\omega_{\mathrm{Ai}}^{2}\right)-\rho_{\mathrm{e}}\left(\omega^{2}-\omega_{\mathrm{Ae}}^{2}\right) \frac{k_{\mathrm{i}}}{k_{\mathrm{e}}} Q_{m} \\
& +\frac{\mathrm{i} \pi k_{z}^{2}}{\rho_{c}\left|\triangle_{c}\right|}\left(\frac{v_{\mathrm{s} c}^{2}}{v_{\mathrm{s} c}^{2}+v_{\mathrm{A} c}^{2}}\right)^{2} \rho_{\mathrm{i}} \rho_{\mathrm{e}}\left(\omega^{2}-\omega_{\mathrm{Ai}}^{2}\right)\left(\omega^{2}-\omega_{\mathrm{Ae}}^{2}\right) \frac{G_{m}}{k_{\mathrm{e}}}=0,
\end{aligned}
$$

where

$G_{m}=\frac{K_{m}\left(k_{\mathrm{e}} R\right)}{K_{m}^{\prime}\left(k_{\mathrm{e}} R\right)}$,

and $D_{m}$ can be written as $D_{m}=D_{m r}+\mathrm{i} D_{m i}$. When there is resonant damping, the wave frequency has real and imaginary parts: $\omega=\omega_{r}+\mathrm{i} \gamma_{m}$ where $\omega_{r}$ is the eigenfrequency of the wave mode. The imaginary part $\gamma_{m}$ can be obtained by $\gamma_{m}=$ $-D_{m i} /\left.\left(\partial D_{m r} / \partial \omega\right)\right|_{\omega=\omega_{r}}$ (e.g. Goossens et al. 1992) by assuming $\left|\gamma_{m}\right| \ll \omega_{r}$. We obtain for $D_{m i}$

$D_{m i}=\frac{\pi \rho_{\mathrm{i}} \rho_{\mathrm{e}} k_{z}^{2}}{k_{\mathrm{e}} \rho_{c}\left|\Delta_{c}\right|}\left(\frac{v_{\mathrm{sc}}^{2}}{v_{\mathrm{sc}}^{2}+v_{\mathrm{A} c}^{2}}\right)^{2}\left(\omega^{2}-\omega_{\mathrm{Ai}}^{2}\right)\left(\omega^{2}-\omega_{\mathrm{Ae}}^{2}\right) G_{m}$,

and $D_{m r}$

$D_{m r}=\rho_{\mathrm{i}}\left(\omega^{2}-\omega_{\mathrm{Ai}}^{2}\right)-\rho_{\mathrm{e}}\left(\omega^{2}-\omega_{\mathrm{Ae}}^{2}\right)\left(\frac{k_{\mathrm{i}}}{k_{\mathrm{e}}}\right) Q_{m}$. 
D. J. Yu et al.: Resonant absorption of the slow sausage wave in the slow continuum

Note that Eq. (30) is the same as Eq. (8).

The analytical formula for $\gamma_{m}$ (see Appendix B) is given as

$$
\begin{array}{r}
\gamma_{m}=\frac{\frac{\pi \rho_{\mathrm{i}} \rho_{\mathrm{e}} k_{z}^{2}}{k_{\mathrm{e}} \rho_{c}\left|\Delta_{c}\right|}\left(\frac{v_{\mathrm{sc}}^{2}}{v_{\mathrm{sc}}^{2}+v_{\mathrm{Ac}}^{2}}\right)^{2}\left(\omega_{r}^{2}-\omega_{\mathrm{Ai}}^{2}\right)\left(\omega_{r}^{2}-\omega_{\mathrm{Ae}}^{2}\right) G_{m}}{\frac{\partial}{\partial \omega_{r}}\left[\rho_{\mathrm{i}}\left(\omega_{r}^{2}-\omega_{\mathrm{Ai}}^{2}\right)-\rho_{\mathrm{e}}\left(\omega_{r}^{2}-\omega_{\mathrm{Ae}}^{2}\right)\left(\frac{k_{\mathrm{i}}}{k_{\mathrm{e}}}\right) Q_{m}\right]} \\
=-\frac{\frac{\pi \rho_{\mathrm{e}} k_{z}^{2}}{k_{\mathrm{e}} \rho_{c} \mid \Delta_{c} l}\left(\frac{v_{\mathrm{sc}}^{2}}{v_{\mathrm{sc}}^{2}+v_{\mathrm{Ac}}^{2}}\right)^{2}\left(\omega_{r}^{2}-\omega_{\mathrm{Ai}}^{2}\right)\left(\omega_{r}^{2}-\omega_{\mathrm{Ae}}^{2}\right) G_{m}}{2 \omega_{r}\left[1-\chi\left(\frac{k_{\mathrm{i}}}{k_{\mathrm{e}}}\right) Q_{m}\right]-\omega_{r} \chi T_{m}},
\end{array}
$$

where

$$
\begin{aligned}
T_{m}= & \omega_{r}^{2}\left(\omega_{r}^{2}-\omega_{\mathrm{Ae}}^{2}\right)\left(\frac{k_{\mathrm{i}}}{k_{\mathrm{e}}}\right)\left\{\frac{\left(\omega_{r}^{2}-2 \omega_{\mathrm{Ci}}^{2}\right)\left[Q_{m}+k_{\mathrm{i}} R P_{m}\right]}{\left(\omega_{r}^{2}-\omega_{\mathrm{si}}^{2}\right)\left(\omega_{r}^{2}-\omega_{\mathrm{Ai}}^{2}\right)\left(\omega_{r}^{2}-\omega_{\mathrm{Ci}}^{2}\right)}\right. \\
& \left.-\frac{\left(\omega_{r}^{2}-2 \omega_{\mathrm{Ce}}^{2}\right)\left[Q_{m}-k_{\mathrm{e}} R S_{m}\right]}{\left(\omega_{r}^{2}-\omega_{\mathrm{se}}^{2}\right)\left(\omega_{r}^{2}-\omega_{\mathrm{Ae}}^{2}\right)\left(\omega_{r}^{2}-\omega_{\mathrm{Ce}}^{2}\right)}\right\} \\
P_{m}= & \left(\frac{I_{m}^{\prime \prime}}{I_{m}}-\frac{I_{m}^{\prime 2}}{I_{m}^{2}}\right) \frac{K_{m}}{K_{m}^{\prime}} \\
S_{m}= & \left(1-\frac{K_{m}^{\prime \prime} K_{m}}{K_{m}^{\prime 2}}\right) \frac{I_{m}^{\prime}}{I_{m}}
\end{aligned}
$$

For the sausage mode $(m=0)$ we obtain,

$\gamma_{0}=-\frac{\pi \rho_{\mathrm{e}} k_{z}^{2}}{k_{\mathrm{e}} \rho_{c}\left|\triangle_{c}\right|}\left(\frac{v_{\mathrm{s} c}^{2}}{v_{\mathrm{s} c}^{2}+v_{\mathrm{A} c}^{2}}\right)^{2} \frac{\left(\omega_{r}^{2}-\omega_{\mathrm{Ai}}^{2}\right)\left(\omega_{r}^{2}-\omega_{\mathrm{Ae}}^{2}\right) G_{0}}{2 \omega_{r}\left[1-\chi\left(\frac{k_{\mathrm{i}}}{k_{\mathrm{e}}}\right) Q_{0}\right]-\omega_{r} \chi T_{0}}$

In the incompressible limit $\left(v_{\mathrm{si}}\left(v_{\mathrm{se}}\right) \rightarrow \infty\right), \gamma_{0}$ reduces to

$\gamma_{0}=-\frac{\pi \rho_{\mathrm{e}} k_{z}^{2}}{k_{\mathrm{e}} \rho_{c}\left|\triangle_{c}\right|} \frac{\left(\omega_{r}^{2}-\omega_{\mathrm{Ai}}^{2}\right)\left(\omega_{r}^{2}-\omega_{\mathrm{Ae}}^{2}\right) G_{0}}{2 \omega_{r}\left[1-\chi Q_{0}\right]}$

since $T_{0}$ goes to zero.

\subsection{Linear profile for the cusp velocity $v_{\mathrm{C}}$}

We are interested in simplified analytical expressions for the damping rate. Therefore, we consider simple profiles for the transitional layer f.e. a linear profile for the cusp velocity $v_{\mathrm{c}}$ in the transitional layer $\left(r_{\mathrm{i}} \leq r \leq r_{\mathrm{e}}\right)$ :

$v_{\mathrm{C}}=v_{\mathrm{Ci}}+\left(v_{\mathrm{Ce}}-v_{\mathrm{Ci}}\right) \frac{r-r_{\mathrm{i}}}{r_{\mathrm{e}}-r_{\mathrm{i}}}$,

where $r_{\mathrm{e}}=R+l / 2$ and $r_{\mathrm{i}}=R-l / 2$.

Then $\triangle_{c}$ becomes

$\Delta_{c}=\mathrm{d}\left(\omega-\omega_{\mathrm{C}}^{2}\right) /\left.\mathrm{d} r\right|_{r=r_{c}}=-2 k_{z}^{2} v_{\mathrm{C}} \frac{\mathrm{d} v_{\mathrm{C}}}{\mathrm{d} r}=-2 \omega_{\mathrm{C}} \frac{\omega_{\mathrm{Ce}}-\omega_{\mathrm{Ci}}}{l}$.

From this formula we can easily see that the damping rate is linearly proportional to $l$.

In the long wavelength limit we obtain

$\Delta_{c} \approx-2 \omega_{\mathrm{Ci}}\left(\omega_{\mathrm{Ce}}-\omega_{\mathrm{Ci}}\right) / l$.

\subsection{Long wavelength limit}

In the limit $k_{\mathrm{i}} R\left(k_{\mathrm{e}} R\right) \ll 1$, the imaginary part $\gamma_{0}$ can be reduced to, by using the asymptotic expansion of $Q_{0}, G_{0}, P_{0}$ and $S_{0}$ (Eqs. (A.5)-(A.8)),

$$
\begin{aligned}
\gamma_{0}= & -\frac{\pi \rho_{\mathrm{i}} \rho_{\mathrm{e}} k_{z}^{2} R}{\rho_{c}\left|\Delta_{c}\right|}\left(\frac{v_{\mathrm{s} c}^{2}}{v_{\mathrm{s} c}^{2}+v_{\mathrm{A} c}^{2}}\right)^{2} \\
& \times \frac{\left(\omega_{r}^{2}-\omega_{\mathrm{Ai}}^{2}\right)\left(\omega_{r}^{2}-\omega_{\mathrm{Ae}}^{2}\right) \ln \left(k_{\mathrm{e}} R\right)}{2 \omega_{r}\left\{\rho_{\mathrm{i}}-\frac{\rho_{\mathrm{e}} k_{\mathrm{i}}^{2} R^{2}}{2} \ln \left(k_{\mathrm{e}} R\right)\right\}-\rho_{\mathrm{e}} \omega_{r} T_{0}},
\end{aligned}
$$

where

$$
\begin{aligned}
T_{0}= & \omega_{r}^{2}\left(\omega_{r}^{2}-\omega_{\mathrm{Ae}}^{2}\right)\left\{\frac{3}{16} \frac{\left(\omega_{r}^{2}-2 \omega_{c i}^{2}\right) k_{\mathrm{i}}^{4} R^{4} \ln \left(k_{\mathrm{e}} R\right)}{\left(\omega_{r}^{2}-\omega_{\mathrm{si}}^{2}\right)\left(\omega_{r}^{2}-\omega_{\mathrm{Ai}}^{2}\right)\left(\omega_{r}^{2}-\omega_{\mathrm{Ci}}^{2}\right)}\right. \\
& \left.+\frac{\left(\omega_{r}^{2}-2 \omega_{\mathrm{Ce}}^{2}\right) k_{\mathrm{i}}^{2} R^{2}}{2\left(\omega_{r}^{2}-\omega_{\mathrm{se}}^{2}\right)\left(\omega_{r}^{2}-\omega_{\mathrm{Ae}}^{2}\right)\left(\omega_{r}^{2}-\omega_{\mathrm{Ce}}^{2}\right)}\right\} .
\end{aligned}
$$

We can further simplify Eq. (40) for $k_{z} R \ll 1$ and $\omega_{r} \approx \omega_{\mathrm{Ci}}$ (using Eqs. (15)-(18)), then Eq. (40) becomes

$\gamma_{0}=\frac{\pi \chi k_{z}^{2} R}{\left|\triangle_{c}\right|}\left(\frac{v_{\mathrm{si}}^{2}}{v_{\mathrm{si}}^{2}+v_{\mathrm{Ai}}^{2}}\right)^{2} \frac{\left(\omega_{\mathrm{Ci}}^{2}-\omega_{\mathrm{Ai}}^{2}\right)\left(\omega_{\mathrm{Ci}}^{2}-\omega_{\mathrm{Ae}}^{2}\right) \ln \left(k_{z} R\right)}{\chi \omega_{\mathrm{Ci}} T_{0}}$,

where

$T_{0}=-\frac{3 \omega_{\mathrm{Ai}}^{8}}{2 \chi^{3} \omega_{\mathrm{si}}^{4}\left(\omega_{\mathrm{Ci}}^{2}-\omega_{\mathrm{Ae}}^{2}\right)^{2} k_{z}^{2} R^{2} \ln ^{2}\left(k_{z} R\right)}$.

Here we have only left a most dominant term in the denominator of the second line in Eq. (40).

For the linear cusp velocity (Eq. (39)), we then obtain

$\gamma_{0}=\frac{\pi \chi^{3}}{3} \frac{l}{R} \frac{\omega_{\mathrm{Ci}}^{3}\left(\omega_{\mathrm{Ci}}^{2}-\omega_{\mathrm{Ae}}^{2}\right)^{3} \omega_{\mathrm{si}}^{2}}{\omega_{\mathrm{Ai}}^{10}}\left(k_{z} R\right)^{4} \ln ^{3}\left(k_{z} R\right)$.

If we consider the photospheric (magnetic pore) conditions where $\omega_{\mathrm{Ae}}\left(\omega_{\mathrm{Ce}}\right) \simeq 0$, Eq. (44) becomes

$\gamma_{0}=\frac{\pi \chi}{3} \frac{l}{R} \frac{\omega_{\mathrm{Ci}}^{9} \omega_{\mathrm{si}}^{2}}{\omega_{\mathrm{Ai}}^{10}}\left(k_{z} R\right)^{4} \ln ^{3}\left(k_{z} R\right)$.

These formulas would be helpful to roughly estimate the damping rate for waves with a long wavelength in the longitudinal direction.

For more detailed derivations of Eqs. (42) and (44) see Appendix C.

\section{Results}

One of our goals is to derive a simple analytical formula for the slow sausage mode in the slow continuum. Another focus is on how strong the effect of resonant damping on the wave is. To estimate this, we have used the parameters $v_{\mathrm{Ae}}=0 \mathrm{~km} \mathrm{~s}^{-1}, v_{\mathrm{Ai}}=$ $12 \mathrm{~km} \mathrm{~s}^{-1}, v_{\mathrm{se}}=11.5 \mathrm{~km} \mathrm{~s}^{-1}, v_{\mathrm{si}}=7 \mathrm{~km} \mathrm{~s}^{-1}, v_{\mathrm{Ce}}=0 \mathrm{~km} \mathrm{~s}^{-1}$, and $v_{\mathrm{Ci}}=6.0464 \mathrm{~km} \mathrm{~s}^{-1}\left(=0.86378 v_{\mathrm{si}}\right)$, which are the same as in previous figures.

We consider the linear cusp velocity profile shown in Sect. 3.3 and show the results in Fig. 3 where $l / R=0.1$. Here we have used formulas Eqs. (35) and (38). The damping rate $\left|\gamma_{0}\right| / \omega_{r}$ shows a quasi-linear behaviour as a function of $k_{z} R$ and 

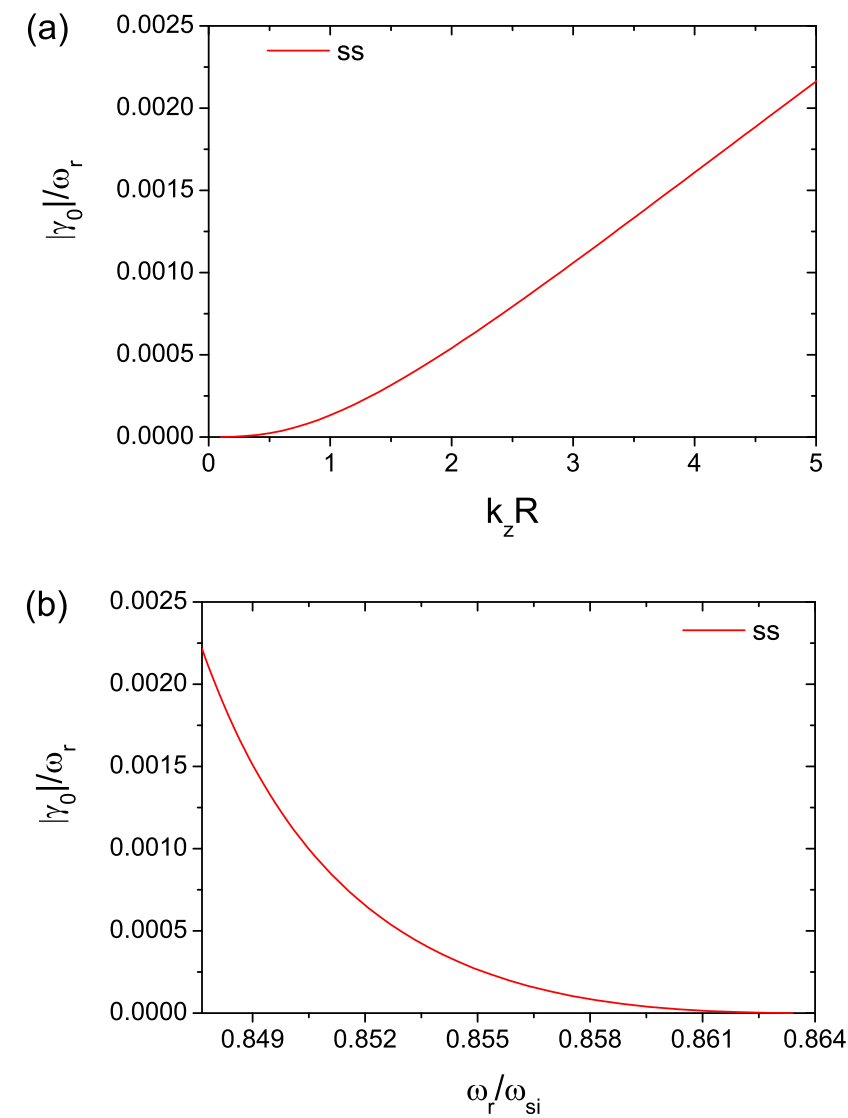

Fig. 3. Damping rate $\left|\gamma_{0}\right| / \omega_{r}$ versus a) $k_{z} R$ and b) $\omega_{r} / \omega_{\mathrm{si}}$ for the slow sausage surface mode (ss) where $l / R=0.1$. The other parameters are the same as in the previous figures. The linear cusp velocity (Eq. (38)) considered in Sect. 3.3 is used.

a decreasing function of $\omega_{r} / \omega_{\mathrm{si}}$, showing that the damping effect due to resonant absorption is very small. For $k_{z} R=5$, $\left|\gamma_{0}\right| / \omega_{r} \approx 0.00216$, and then the ratio of damping time to the period $\tau_{\mathrm{D}} / T$ is $\left(1 /\left|\gamma_{0}\right|\right) /\left(2 \pi / \omega_{r}\right)=1 /\left(2 \pi\left|\gamma_{0}\right| / \omega_{r}\right) \approx 73.6$. This value is quite big compared to the typical value for resonant damping of the kink mode, which is approximately 2-4 (e.g. Goossens et al. 2002).

In Fig. 4 we show $l / R$-dependent behaviour of (a) the damping rate $\left|\gamma_{0}\right| / \omega_{r}$ and (b) the ratio of damping time to the period $\tau_{\mathrm{D}} / T$ for different $k_{z} R$ values. The other parameters are the same as in previous results. Since the wave frequency of slow surface mode depends on $k_{z} R$, we denote the corresponding wave frequency for each $k_{z} R$ in panel a of the figure. The damping rate $\left|\gamma_{0}\right| / \omega_{r}$ increases as $k_{z} R$ and $l / R$ increase. The ratio of damping time to the period $\tau_{\mathrm{D}} / T$, as a result, decreases as $k_{z} R$ and $l / R$ increase. For example, when $k_{z} R=5$ and $l / R=0.5, \tau_{\mathrm{D}} / T \approx 14.7$, which is comparable to, but still larger than the corresponding value for the kink mode.

In Sect. 3.4, we have derived a simple formula for the damping rate in the long wavelength limit where $\omega \approx \omega_{\mathrm{Ci}}$. In Fig. 5, we compare this formula, Eq. (45), with the analytical formula, Eq. (35) (panel a in Fig. 3) for the same linear cusp velocity. The figure shows that the long wavelength limit approximation overestimates the damping rate when $k_{z} R<0.354$ and underestimates it when $k_{z} R>0.354$, resulting in convergence when $k_{z} R$ goes to zero. The valid range of the long wavelength limit approximation is small. The difference between two formulas becomes larger as $k_{z} R$ increases from 0.354 . So, the long wavelength approximation leads to an underestimation under
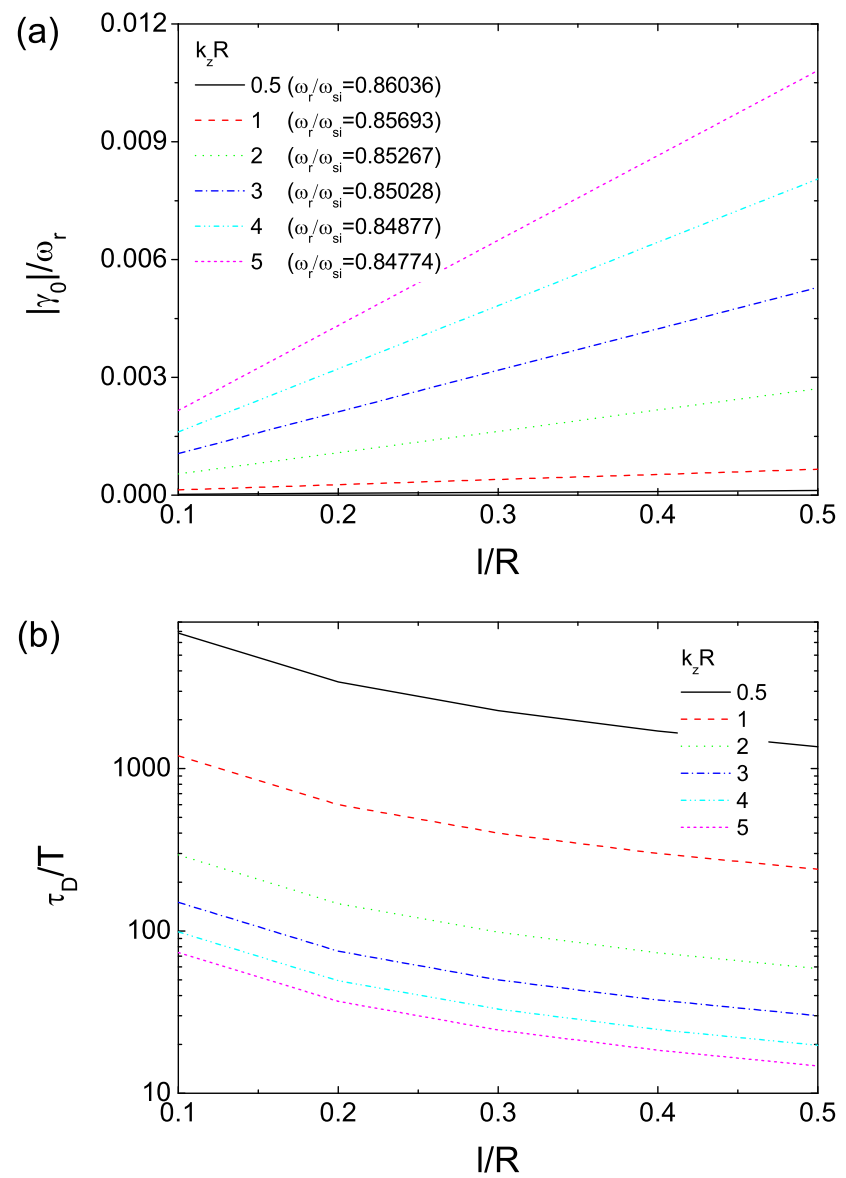

Fig. 4. a) Damping rate $\left|\gamma_{0}\right| / \omega_{r}$ and b) the ratio of damping time to the period $\tau_{\mathrm{D}} / T$ versus $l / R$. For the slow surface mode (ss). The other parameters are the same as in the previous figures. In a) we denote the value of the wave frequency $\left(\omega_{r} / \omega_{\mathrm{si}}\right)$ corresponding to each value of $k_{z} R$, which is omitted in b). In b) $\tau_{\mathrm{D}} / T$ is shown in log scale.

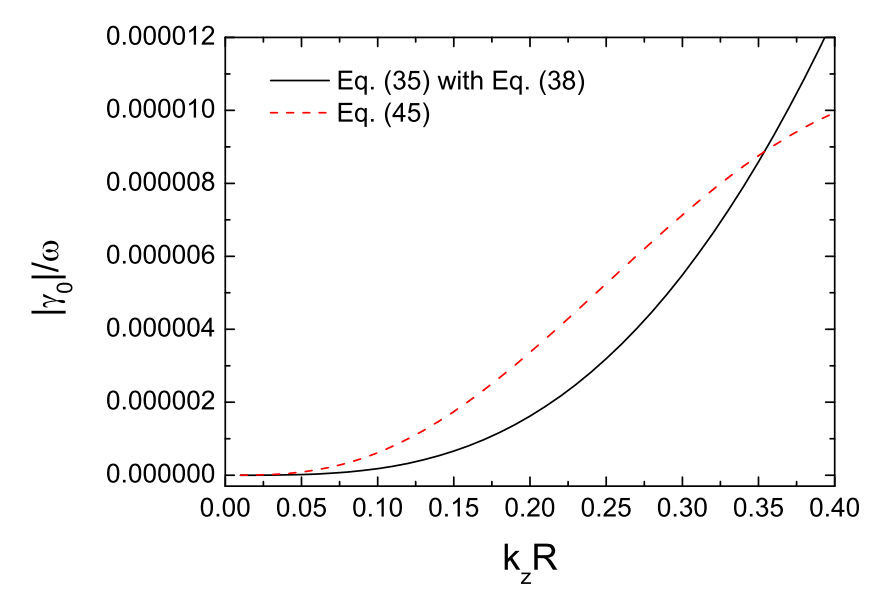

Fig. 5. Damping rate $\left|\gamma_{0}\right| / \omega$ versus $k_{z} R$ for the slow sausage surface mode (ss) where $l / R=0.1$. The other parameters are the same as in the previous figures. For the linear cusp velocity, we compare the analytical formula, Eq. (35), with the simplified formula in the long wavelength limit, Eq. (45).

magnetic pore conditions. Note that Eq. (45) has no dependency on the frequency; the frequency is fixed as $\omega_{\mathrm{Ci}}$, while Eq. (35) has a frequency dependence $\left(\omega=\omega_{r}\right)$.

The assumption of a linear cusp velocity in the transitional layer is a rough treatment for the resonant damping. In general, the structure of the transitional layer is more complicated. We 
need to consider certain profiles for density, magnetic field, and pressure, which should satisfy the condition for an equilibrium. By considering the linear density profile and linear pressure profile in the inhomogeneous layer, we have found that the damping rate increases by a factor of $\approx 10$ compared to that for the linear cusp velocity. We will show the detailed results for this configuration in a future paper.

\section{Summary and discussions}

We have derived a new (to the best of our knowledge) general analytical formula (Eq. (35)) for the damping rate of the slow surface sausage mode in the slow continuum by considering the thin boundary (TB) approximation. Although we have focused on the analytical solution Eq. (35) with Eq. (10) for the damping rate of the slow surface sausage mode, we point out that Eq. (31) is a general formula applicable to any kind of surface modes (any $m$ values). If we change the modified Bessel function $I_{m}$ into Bessel function $J_{m}$ in Sect. 2 we also obtain the analytical formula for the body modes with arbitrary $m$ (not shown in the paper) provided that the frequency of the body mode is in the slow continuum. The same procedure adopted in this paper has been applied before to the wave damping by resonant absorption for $m>0$ (see e.g. Sakurai et al. 1991; Goossens et al. 1992).

By considering a linear cusp velocity in the transitional layer and the long wavelength limit for the surface mode under photospheric conditions, we have obtained simple analytical formulas, Eqs. (44) and (45), which can be useful to roughly estimate the wave damping due to resonant absorption for the wave with long longitudinal wavelength. We have compared this simplified version with our former analytical formula. The difference between the two formulas is relatively small when the longitudinal wavelength is long.

We have applied the analytical formula to the observational rapid damping of slow surface mode in Grant et al. (2015). For the conventional magnetic pore $R \approx 0.5-3 \mathrm{Mm}$ and $k_{z}=2 \pi / \lambda_{z}=$ $2 \pi / 4400 \mathrm{~km}$, which yields $k_{z} R \approx 0.7-4.3$. For $k_{z} R=4.3$ and $l / R=0.5$, the assumption of a linear cusp velocity (Eq. (38)) gives $\left|\gamma_{0}\right| / \omega_{r}=0.0089$. Then the ratio of damping time to wave period is approximately 17.9. This value can be further reduced if we consider linear density and linear pressure profiles in the transitional layer as mentioned in the previous section. This result implies that the resonant absorption in the slow continuum could be efficient for the damping of the slow sausage waves in the lower solar atmosphere, although the damping of the sausage mode in the slow continuum is not as extremely rapid as that of the kink mode in the Alfvén continuum. In previous studies, the role of the slow resonance in wave damping for $m>0$ was considered to be negligible compared to the Alfvén resonance due to the factor $\left(\tau_{D \mathrm{~A}} / \tau_{D \mathrm{~s}}\right) \approx\left(k_{z} R / m\right)^{2}\left(v_{\mathrm{s}}^{2} /\left(v_{\mathrm{s}}^{2}+v_{\mathrm{A}}^{2}\right)\right)^{2}$, which is very small in the upper solar atmosphere (Soler et al. 2009), where the subscripts A and s denote the Alfvén and slow resonances. Our results also signify that the damping rate is crucially dependent on the density, pressure, and magnetic field profiles. On the contrary, the obtained damping periods are too long to explain the rapid decay of the wave energy flux in Grant et al. (2015). The resonant absorption mechanism is not very important in this case, but it could play a crucial role in other observations of slow sausage wave damping.

Our theory is based on the assumption that the magnetic flux tube maintains its shape with only a small perturbation during the wave propagation and associated resonant absorption, so the practical effect on the wave damping would be below the theoretical estimate. Expanding magnetic flux structure of sunspots and magnetic pores restricts the range of validity of modelling it as a straight cylinder. Extension to a more general magnetic field configuration is necessary.

Whilst we have considered a straight magnetic field along the cylindrical tube, the inclusion of azimuthal field makes it possible for both Alfvén and slow resonance continua to overlap in some frequency regime. Although it is complicated and difficult to analytically study, resonant absorption of the sausage modes in both continua may give intriguing results as, for example, Giagkiozis et al. (2016) have shown that the longitudinal field and density is of importance when considering the damping rate of sausage modes in the Alfvén continuum. This issue will be pursued in the future.

Acknowledgements. This work was supported by the Odysseus type II funding (FWO-Vlaanderen), IAP P7/08 CHARM (Belspo), and GOA-2015-014 (KU Leuven)

\section{References}

Abramowitz, M., \& Stegun, I. A. 1970, Handbook of mathematical functions: with formulas, graphs, and mathematical tables (New York: Dover) Arregui, I., Soler, R., Ballester, J. L., \& Wright, A. N. 2011, A\&A, 533, A60 Cadez, V. M., Csik, A., Erdelyi, R., \& Goossens, M. 1997, A\&A, 326, 1241 De Moortel, I., \& Hood, A. W. 2003, A\&A, 408, 755

De Moortel, I., \& Hood, A. W. 2004, A\&A, 415, 705

Dorotovič, I., Erdélyi, R., \& Karlovský, V. 2008, in Waves Oscillations in the Solar Atmosphere: Heating and Magneto-Seismology, eds. R. Erdélyi, \& C. A. Mendoza-Briceno, IAU Symp., 247, 351

Dorotovič, I., Erdélyi, R., Freij, N., Karlovský, V., \& Márquez, I. 2014, A\&A, 563, A12

Edwin, P. M., \& Roberts, B. 1983, Sol. Phys., 88, 179

Evans, D. J., \& Roberts, B. 1990, ApJ, 348, 346

Freij, N., Dorotovič, I., Morton, R. J., et al. 2016, ApJ, 817, 44

Fujimura, D., \& Tsuneta, S. 2009, ApJ, 702, 1443

Giagkiozis, I., Goossens, M., Verth, G., Fedun, V., \& Van Doorsselaere, T. 2016, ApJ, 823, 71

Goossens, M., Hollweg, J. V., \& Sakurai, T. 1992, Sol. Phys., 138, 233

Goossens, M., Andries, J., \& Aschwanden, M. J. 2002, A\&A, 394, L39

Goossens, M., Arregui, I., Ballester, J. L., \& Wang, T. J. 2008, A\&A, 484, 851

Grant, S. D. T., Jess, D. B., Moreels, M. G., et al. 2015, ApJ, 806, 132

Kanoh, R., Shimizu, T., \& Imada, S. 2016, ApJ, 831, 24

Keppens, R. 1995, Sol. Phys., 161, 251

Keppens, R. 1996, ApJ, 468, 907

Krishna Prasad, S., Banerjee, D., \& Van Doorsselaere, T. 2014, ApJ, 789, 118

Mandal, S., Magyar, N., Yuan, D., Van Doorsselaere, T., \& Banerjee, D. 2016, ApJ, 820, 13

Moreels, M. G., \& Van Doorsselaere, T. 2013, A\&A, 551, A137

Moreels, M. G., Goossens, M., \& Van Doorsselaere, T. 2013, A\&A, 555, A75

Moreels, M. G., Freij, N., Erdélyi, R., Van Doorsselaere, T., \& Verth, G. 2015a, A\&A, 579, A73

Moreels, M. G., Van Doorsselaere, T., Grant, S. D. T., Jess, D. B., \& Goossens, M. 2015b, A\&A, 578, A60

Morton, R. J., Erdélyi, R., Jess, D. B., \& Mathioudakis, M. 2011, ApJ, 729, L18 Ruderman, M. S. 2009, Phys. Plasmas, 16, 042109

Ruderman, M. S., Oliver, R., Erdélyi, R., Ballester, J. L., \& Goossens, M. 2000 A\&A, 354, 261

Sakurai, T., Goossens, M., \& Hollweg, J. V. 1991, Sol. Phys., 133, 227

Soler, R., Oliver, R., Ballester, J. L., \& Goossens, M. 2009, ApJ, 695, L166

Soler, R., Goossens, M., Terradas, J., \& Oliver, R. 2013, ApJ, 777, 158

Terradas, J., Oliver, R., \& Ballester, J. L. 2006, ApJ, 642, 533

Van Doorsselaere, T., Andries, J., Poedts, S., \& Goossens, M. 2004, ApJ, 606, 1223

Yu, D. J., \& Van Doorsselaere, T. 2016, ApJ, 831, 30 


\section{Appendix A: Asymptotic values of $Q_{m}, G_{m}, P_{m}$ and $S_{m}$}

We only show the case of $m=0$, but similar procedures apply to other $m$ modes (e.g. Abramowitz \& Stegun 1970).

\section{A.1. Surface mode}

For the surface mode with $m=0$ we have

$$
\begin{aligned}
Q_{0} & =\left[\frac{I_{0}^{\prime}\left(k_{\mathrm{i}} R\right) K_{0}\left(k_{\mathrm{e}} R\right)}{I_{0}\left(k_{\mathrm{i}} R\right) K_{0}^{\prime}\left(k_{\mathrm{e}} R\right)}\right]=\left(\frac{I_{1}}{I_{0}}\right)\left(\frac{K_{0}}{-K_{1}}\right)=-\left(\frac{I_{1}}{I_{0}}\right)\left(\frac{K_{0}}{K_{1}}\right), \\
G_{0} & =\left[\frac{K_{0}\left(k_{\mathrm{e}} R\right)}{K_{0}^{\prime}\left(k_{\mathrm{e}} R\right)}\right]=\left(\frac{K_{0}}{-K_{1}}\right)=-\left(\frac{K_{0}}{K_{1}}\right), \\
P_{0} & =\left(\frac{I_{0}^{\prime \prime}}{I_{0}}-\frac{I_{0}^{\prime 2}}{I_{0}^{2}}\right) \frac{K_{0}}{K_{0}^{\prime}}=\left(\frac{1}{2} \frac{I_{0}+I_{2}}{I_{0}}-\frac{I_{1}^{2}}{I_{0}^{2}}\right)\left(\frac{K_{0}}{-K_{1}}\right) \\
& =-\left(\frac{1}{2}+\frac{I_{2}}{2 I_{0}}-\frac{I_{1}^{2}}{I_{0}^{2}}\right) \frac{K_{0}}{K_{1}}, \\
S_{0} & =\left(1-\frac{K_{0}^{\prime \prime} K_{0}}{K_{0}^{\prime 2}}\right) \frac{I_{0}^{\prime}}{I_{0}}=\left(1-\frac{-K_{1}^{\prime} K_{0}}{K_{1}^{2}}\right) \frac{I_{1}}{I_{0}} \\
& =\left(1-\frac{1}{2} \frac{\left(K_{0}+K_{2}\right) K_{0}}{K_{1}^{2}}\right) \frac{I_{1}}{I_{0}} .
\end{aligned}
$$

For the case $k_{\mathrm{i}} r_{c}\left(k_{\mathrm{e}} r_{c}\right)<1$ (first order approximation) we derive

$$
\begin{aligned}
Q_{0} & =\left(\frac{I_{1}}{I_{0}}\right)\left(\frac{K_{0}}{-K_{1}}\right) \approx\left(\frac{k_{\mathrm{i}} R / 2}{1}\right)\left(\frac{-\ln \left(k_{\mathrm{e}} R / 2\right)-\gamma_{\mathrm{e}}}{-1 / k_{\mathrm{e}} R}\right) \\
& =\frac{k_{\mathrm{i}} k_{\mathrm{e}} R^{2}}{2}\left[\ln \left(\frac{k_{\mathrm{e}} R}{2}\right)+\gamma_{\mathrm{e}}\right], \\
G_{0} & =\left(\frac{K_{0}}{-K_{1}}\right) \approx\left(\frac{-\ln \left(k_{\mathrm{e}} R / 2\right)-\gamma_{\mathrm{e}}}{-1 / k_{\mathrm{e}} R}\right) \\
& =k_{\mathrm{e}} R\left[\ln \left(\frac{k_{\mathrm{e}} R}{2}\right)+\gamma_{\mathrm{e}}\right], \\
P_{0} & =-\left(\frac{1}{2}+\frac{I_{2}}{2 I_{0}}-\frac{I_{1}^{2}}{I_{0}^{2}}\right) \frac{K_{0}}{K_{1}} \\
& \approx-\left(\frac{1}{2}+\frac{\left(k_{\mathrm{i}} R\right)^{2} / 8}{2}-\frac{\left(k_{\mathrm{i}} R / 2\right)^{2}}{1}\right)\left(\frac{-\ln \left(k_{\mathrm{e}} R / 2\right)-\gamma_{\mathrm{e}}}{-1 / k_{\mathrm{e}} R}\right) \\
& =-k_{\mathrm{e}} R\left(\frac{1}{2}-\frac{3}{16}\left(k_{\mathrm{i}} R\right)^{2}\right)\left[\ln \left(\frac{k_{\mathrm{e}} R}{2}\right)+\gamma_{\mathrm{e}}\right],
\end{aligned}
$$

$$
\begin{aligned}
S_{0}= & \left(1-\frac{1}{2} \frac{\left(K_{0}+K_{2}\right) K_{0}}{K_{1}^{2}}\right) \frac{I_{1}}{I_{0}} \approx\left[1-\frac{1}{2}\left(\frac{-\ln \left(k_{\mathrm{e}} R / 2\right)-\gamma_{\mathrm{e}}}{-1 / k_{\mathrm{e}} R}\right)^{2}\right. \\
& \left.-\frac{1}{2} \frac{\left(2 /\left(k_{\mathrm{e}} R\right)^{2}\right)\left(-\ln \left(k_{\mathrm{e}} R / 2\right)-\gamma_{\mathrm{e}}\right)}{\left(-1 / k_{\mathrm{e}} R\right)^{2}}\right]\left(\frac{k_{\mathrm{i}} R / 2}{1}\right) \\
= & \left(1-\frac{\left(k_{\mathrm{e}} R\right)^{2}\left[\ln \left(k_{\mathrm{e}} R / 2\right)+\gamma_{\mathrm{e}}\right]^{2}}{2}+\ln \left(k_{\mathrm{e}} R / 2\right)+\gamma_{\mathrm{e}}\right)\left(\frac{k_{\mathrm{i}} R}{2}\right) \\
= & \frac{k_{\mathrm{i}} R}{2}\left\{1+\left[\ln \left(\frac{k_{\mathrm{e}} R}{2}\right)+\gamma_{\mathrm{e}}\right]-\frac{\left(k_{\mathrm{e}} R\right)^{2}}{2}\left[\ln \left(\frac{k_{\mathrm{e}} R}{2}\right)+\gamma_{\mathrm{e}}\right]^{2}\right\} \\
\approx & \frac{k_{\mathrm{i}} R}{2}\left\{1+\left[\ln \left(\frac{k_{\mathrm{e}} R}{2}\right)+\gamma_{\mathrm{e}}\right]\right\},
\end{aligned}
$$

where $\gamma_{\mathrm{e}}$ is the Euler's constant.

A108, page 8 of 10

\section{A.2. Body mode}

For the body modes with $m=0$ we have

$$
\begin{aligned}
Q_{0} & =\left[\frac{J_{0}^{\prime}\left(k_{\mathrm{i}} R\right) K_{0}\left(k_{\mathrm{e}} R\right)}{J_{0}\left(k_{\mathrm{i}} R\right) K_{0}^{\prime}\left(k_{\mathrm{e}} R\right)}\right]=\left(\frac{-J_{1}}{J_{0}}\right)\left(\frac{K_{0}}{-K_{1}}\right)=\left(\frac{J_{1}}{J_{0}}\right)\left(\frac{K_{0}}{K_{1}}\right) \\
G_{0} & =\left[\frac{K_{0}\left(k_{\mathrm{e}} R\right)}{K_{0}^{\prime}\left(k_{\mathrm{e}} R\right)}\right]=\left(\frac{K_{0}}{-K_{1}}\right)=-\left(\frac{K_{0}}{K_{1}}\right) \\
P_{0} & =\left(\frac{J_{0}^{\prime \prime}}{J_{0}}-\frac{J_{0}^{\prime 2}}{J_{0}^{2}}\right) \frac{K_{0}}{K_{0}^{\prime}} \\
& =\left(\frac{1}{2} \frac{-J_{0}+J_{2}}{J_{0}}-\frac{J_{1}^{2}}{J_{0}^{2}}\right)\left(\frac{K_{0}}{-K_{1}}\right)=-\left(-\frac{1}{2}+\frac{J_{2}}{2 J_{0}}-\frac{J_{1}^{2}}{J_{0}^{2}}\right) \frac{K_{0}}{K_{1}} \\
S_{0} & =\left(1-\frac{K_{0}^{\prime \prime} K_{0}}{K_{0}^{\prime 2}}\right) \frac{J_{0}^{\prime}}{J_{0}}=\left(1-\frac{-K_{1}^{\prime} K_{0}}{K_{1}^{2}}\right) \frac{-J_{1}}{J_{0}} \\
& =-\left(1-\frac{1}{2} \frac{\left(K_{0}+K_{2}\right) K_{0}}{K_{1}^{2}}\right) \frac{J_{1}}{J_{0}}
\end{aligned}
$$

For the case $k_{\mathrm{i}} R\left(k_{\mathrm{e}} R\right)<1$ (first order approximation) we derive

$$
\begin{aligned}
Q_{0} & =\left(\frac{-J_{1}}{J_{0}}\right)\left(\frac{K_{0}}{-K_{1}}\right) \approx\left(-\frac{k_{\mathrm{i}} r_{c} / 2}{1}\right)\left(\frac{-\ln \left(k_{\mathrm{e}} R / 2\right)-\gamma_{\mathrm{e}}}{-1 / k_{\mathrm{e}} R}\right) \\
& =-\frac{k_{\mathrm{i}} k_{\mathrm{e}} R^{2}}{2}\left[\ln \left(\frac{k_{\mathrm{e}} R}{2}\right)+\gamma_{\mathrm{e}}\right],
\end{aligned}
$$

$$
\begin{aligned}
G_{0} & =\left(\frac{K_{0}}{-K_{1}}\right) \approx\left(\frac{-\ln \left(k_{\mathrm{e}} R / 2\right)-\gamma_{\mathrm{e}}}{-1 / k_{\mathrm{e}} R}\right) \\
& =k_{\mathrm{e}} R\left[\ln \left(\frac{k_{\mathrm{e}} R}{2}\right)+\gamma_{\mathrm{e}}\right], \\
P_{0} & =-\left(-\frac{1}{2}+\frac{J_{2}}{2 J_{0}}-\frac{J_{1}^{2}}{J_{0}^{2}}\right) \frac{K_{0}}{K_{1}} \\
& \approx-\left(-\frac{1}{2}+\frac{\left(k_{\mathrm{i}} R\right)^{2} / 8}{2}-\frac{\left(k_{\mathrm{i}} R / 2\right)^{2}}{1}\right)\left(\frac{-\ln \left(k_{\mathrm{e}} R / 2\right)-\gamma_{\mathrm{e}}}{-1 / k_{\mathrm{e}} R}\right) \\
& =-k_{\mathrm{e}} R\left(-\frac{1}{2}-\frac{3}{16}\left(k_{\mathrm{i}} R\right)^{2}\right)\left[\ln \left(\frac{k_{\mathrm{e}} R}{2}\right)+\gamma_{\mathrm{e}}\right] \\
& \approx \frac{k_{\mathrm{e}} R}{2}\left[\ln \left(\frac{k_{\mathrm{e}} R}{2}\right)+\gamma_{\mathrm{e}}\right],
\end{aligned}
$$$$
S_{0}=-\left(1-\frac{1}{2} \frac{\left(K_{0}+K_{2}\right) K_{0}}{K_{1}^{2}}\right) \frac{J_{1}}{J_{0}} \approx-\left[1-\frac{1}{2}\left(\frac{-\ln \left(k_{\mathrm{e}} R / 2\right)-\gamma_{\mathrm{e}}}{-1 / k_{\mathrm{e}} R}\right)^{2}\right.
$$$$
\left.-\frac{1}{2} \frac{\left(2 /\left(k_{\mathrm{e}} R\right)^{2}\right)\left(-\ln \left(k_{\mathrm{e}} R / 2\right)-\gamma_{\mathrm{e}}\right)}{\left(-1 / k_{\mathrm{e}} R\right)^{2}}\right]\left(\frac{k_{\mathrm{i}} R / 2}{1}\right)
$$$$
=-\left(1-\frac{\left(k_{\mathrm{e}} R\right)^{2}\left[\ln \left(k_{\mathrm{e}} R / 2\right)+\gamma_{\mathrm{e}}\right]^{2}}{2}+\ln \left(k_{\mathrm{e}} R / 2\right)+\gamma_{\mathrm{e}}\right)\left(\frac{k_{\mathrm{i}} R}{2}\right)
$$$$
=-\frac{k_{\mathrm{i}} R}{2}\left\{1+\left[\ln \left(\frac{k_{\mathrm{e}} R}{2}\right)+\gamma_{\mathrm{e}}\right]-\frac{\left(k_{\mathrm{e}} R\right)^{2}}{2}\left[\ln \left(\frac{k_{\mathrm{e}} R}{2}\right)+\gamma_{\mathrm{e}}\right]^{2}\right\}
$$$$
\approx-\frac{k_{\mathrm{i}} R}{2}\left\{1+\left[\ln \left(\frac{k_{\mathrm{e}} R}{2}\right)+\gamma_{\mathrm{e}}\right]\right\} \text {. }
$$

\section{Appendix B: Damping rate for the surface mode}

In order to calculate $\gamma_{m}$, we need to derive the expression for $\partial D_{m r} / \partial \omega$ where $\omega$ should be in the slow (cusp) continuum. We 
D. J. Yu et al.: Resonant absorption of the slow sausage wave in the slow continuum

have

$$
\begin{aligned}
\frac{\partial D_{m r}}{\partial \omega}= & 2 \rho_{\mathrm{i}} \omega-2 \omega \rho_{\mathrm{e}}\left(\frac{k_{\mathrm{i}}}{k_{\mathrm{e}}}\right) Q_{m} \\
& -\rho_{\mathrm{e}}\left(\omega^{2}-\omega_{\mathrm{Ae}}^{2}\right)\left(\frac{1}{k_{\mathrm{e}}} \frac{\mathrm{d} k_{\mathrm{i}}}{\mathrm{d} \omega}-\frac{k_{\mathrm{i}}}{k_{\mathrm{e}}^{2}} \frac{\mathrm{d} k_{\mathrm{e}}}{\mathrm{d} \omega}\right) Q_{m} \\
& -\rho_{\mathrm{e}}\left(\omega^{2}-\omega_{\mathrm{Ae}}^{2}\right)\left(\frac{k_{\mathrm{i}}}{k_{\mathrm{e}}}\right) \frac{\mathrm{d} Q_{m}}{\mathrm{~d} \omega} .
\end{aligned}
$$

For $\left(\mathrm{d} k_{\mathrm{i}} / \mathrm{d} \omega\right)$ and $\left(\mathrm{d} k_{\mathrm{e}} / \mathrm{d} \omega\right)$ we derive

$$
\begin{aligned}
\left(\frac{\mathrm{d} k_{\mathrm{i}}}{\mathrm{d} \omega}\right)= & \left(\frac{1}{2 k_{\mathrm{i}}}\right)\left(\frac{1}{v_{\mathrm{si}}^{2}+v_{\mathrm{Ai}}^{2}}\right)\left\{\frac{2 \omega\left[\omega_{\mathrm{Ai}}^{2}-2 \omega^{2}+\omega_{\mathrm{si}}^{2}\right]\left(\omega^{2}-\omega_{\mathrm{Ci}}^{2}\right)}{\left(\omega^{2}-\omega_{\mathrm{Ci}}^{2}\right)^{2}}\right. \\
& \left.-\frac{2 \omega\left(\omega^{2}-\omega_{\mathrm{si}}^{2}\right)\left(\omega_{\mathrm{Ai}}^{2}-\omega^{2}\right)}{\left(\omega^{2}-\omega_{\mathrm{Ci}}^{2}\right)^{2}}\right\} \\
= & -\frac{\omega^{3}}{v_{\mathrm{si}}^{2}+v_{\mathrm{Ai}}^{2}} \frac{\left(\omega^{2}-2 \omega_{\mathrm{Ci}}^{2}\right)}{\left(\omega^{2}-\omega_{\mathrm{Ci}}^{2}\right)^{2} k_{\mathrm{i}}}
\end{aligned}
$$

and, in the same way,

$\left(\frac{\mathrm{d} k_{\mathrm{e}}}{\mathrm{d} \omega}\right)=-\frac{\omega^{3}}{v_{\mathrm{Se}}^{2}+v_{\mathrm{Ae}}^{2}} \frac{\left(\omega^{2}-2 \omega_{\mathrm{Ce}}^{2}\right)}{\left(\omega^{2}-\omega_{\mathrm{Ce}}^{2}\right)^{2} k_{\mathrm{e}}}$.

For $\mathrm{d} Q_{m} / \mathrm{d} \omega$ we obtain

$$
\begin{aligned}
\frac{\mathrm{d} Q_{m}}{\mathrm{~d} \omega}= & \frac{\mathrm{d}}{\mathrm{d} \omega}\left[\frac{I_{m}^{\prime}\left(k_{\mathrm{i}} R\right) K_{m}\left(k_{\mathrm{e}} R\right)}{I_{m}\left(k_{\mathrm{i}} R\right) K_{m}^{\prime}\left(k_{\mathrm{e}} R\right)}\right] \\
= & \frac{1}{I_{m}^{2}\left(k_{\mathrm{i}} R\right) K_{m}^{\prime 2}\left(k_{\mathrm{e}} R\right)}\left\{\frac{\mathrm{d}\left[I_{m}^{\prime}\left(k_{\mathrm{i}} R\right) K_{m}\left(k_{\mathrm{e}} R\right)\right]}{\mathrm{d} \omega} I_{m}\left(k_{\mathrm{i}} R\right) K_{m}^{\prime}\left(k_{\mathrm{e}} R\right)\right. \\
& \left.-I_{m}^{\prime}\left(k_{\mathrm{i}} R\right) K_{m}\left(k_{\mathrm{e}} R\right) \frac{\mathrm{d}\left[I_{m}\left(k_{\mathrm{i}} R\right) K_{m}^{\prime}\left(k_{\mathrm{e}} R\right)\right]}{\mathrm{d} \omega}\right\} \\
= & R\left(\frac{I_{m}^{\prime \prime}}{I_{m}}-\frac{I_{m}^{\prime 2}}{I_{m}^{2}}\right) \frac{K_{m}}{K_{m}^{\prime}} \frac{\mathrm{d} k_{\mathrm{i}}}{\mathrm{d} \omega}+R\left(1-\frac{K_{m}^{\prime \prime} K_{m}}{K_{m}^{\prime 2}}\right) \frac{I_{m}^{\prime}}{I_{m}} \frac{\mathrm{d} k_{\mathrm{e}}}{\mathrm{d} \omega}
\end{aligned}
$$

where the prime means the derivative with respect to the entire argument.

By means of Eqs. (B.2) and (B.3), Eq. (B.4) becomes

$$
\begin{aligned}
\frac{\mathrm{d} Q_{m}}{\mathrm{~d} \omega}= & R\left(\frac{I_{m}^{\prime \prime}}{I_{m}}-\frac{I_{m}^{\prime 2}}{I_{m}^{2}}\right) \frac{K_{m}}{K_{m}^{\prime}} \frac{\mathrm{d} k_{\mathrm{i}}}{\mathrm{d} \omega}+R\left(1-\frac{K_{m}^{\prime \prime} K_{m}}{K_{m}^{\prime 2}}\right) \frac{I_{m}^{\prime}}{I_{m}} \frac{\mathrm{d} k_{\mathrm{e}}}{\mathrm{d} \omega} \\
= & k_{\mathrm{i}} R\left(\frac{I_{m}^{\prime \prime}}{I_{m}}-\frac{I_{m}^{\prime 2}}{I_{m}^{2}}\right) \frac{K_{m}}{K_{m}^{\prime}} \frac{\omega^{3}\left(\omega^{2}-2 \omega_{\mathrm{Ci}}^{2}\right)}{\left(\omega^{2}-\omega_{\mathrm{si}}^{2}\right)\left(\omega^{2}-\omega_{\mathrm{Ai}}^{2}\right)\left(\omega^{2}-\omega_{\mathrm{Ci}}^{2}\right)} \\
& +k_{\mathrm{e}} R\left(1-\frac{K_{m}^{\prime \prime} K_{m}}{K_{m}^{\prime 2}}\right) \frac{I_{m}^{\prime}}{I_{m}} \frac{\omega^{3}\left(\omega^{2}-2 \omega_{\mathrm{Ce}}^{2}\right)}{\left(\omega^{2}-\omega_{\mathrm{se}}^{2}\right)\left(\omega^{2}-\omega_{\mathrm{Ae}}^{2}\right)\left(\omega^{2}-\omega_{\mathrm{Ce}}^{2}\right)} \\
= & k_{\mathrm{i}} R P_{m} \frac{\omega^{3}\left(\omega^{2}-2 \omega_{\mathrm{Ci}}^{2}\right)}{\left(\omega^{2}-\omega_{\mathrm{si}}^{2}\right)\left(\omega^{2}-\omega_{\mathrm{Ai}}^{2}\right)\left(\omega^{2}-\omega_{\mathrm{Ci}}^{2}\right)} \\
& +k_{\mathrm{e}} R S_{m} \frac{\omega^{3}\left(\omega^{2}-2 \omega_{\mathrm{Ce}}^{2}\right)}{\left(\omega^{2}-\omega_{\mathrm{se}}^{2}\right)\left(\omega^{2}-\omega_{\mathrm{Ae}}^{2}\right)\left(\omega^{2}-\omega_{\mathrm{Ce}}^{2}\right)}
\end{aligned}
$$

where $P_{m}$ and $S_{m}$ are

$$
\begin{aligned}
P_{m} & =\left(\frac{I_{m}^{\prime \prime}}{I_{m}}-\frac{I_{m}^{2}}{I_{m}^{2}}\right) \frac{K_{m}}{K_{m}^{\prime}}, \\
S_{m} & =\left(1-\frac{K_{m}^{\prime \prime} K_{m}}{K_{m}^{\prime 2}}\right) \frac{I_{m}^{\prime}}{I_{m}} .
\end{aligned}
$$

Using Eqs. (B.2), (B.3) and (B.5) we have for $\partial D_{m r} / \partial \omega$ $\left(=\mathrm{d} D_{m r} / \mathrm{d} \omega\right)$

$$
\begin{aligned}
& \frac{\mathrm{d} D_{m r}}{\mathrm{~d} \omega}=2 \rho_{\mathrm{i}} \omega-2 \omega \rho_{\mathrm{e}}\left(\frac{k_{\mathrm{i}}}{k_{\mathrm{e}}}\right) Q_{m} \\
& -\rho_{\mathrm{e}}\left(\omega^{2}-\omega_{\mathrm{Ae}}^{2}\right)\left(\frac{1}{k_{\mathrm{e}}} \frac{\mathrm{d} k_{\mathrm{i}}}{\mathrm{d} \omega}-\frac{k_{\mathrm{i}}}{k_{\mathrm{e}}^{2}} \frac{\mathrm{d} k_{\mathrm{e}}}{\mathrm{d} \omega}\right) Q_{m} \\
& -\rho_{\mathrm{e}}\left(\omega^{2}-\omega_{\mathrm{Ae}}^{2}\right)\left(\frac{k_{\mathrm{i}}}{k_{\mathrm{e}}}\right) \frac{\mathrm{d} Q_{m}}{\mathrm{~d} \omega} \\
& =2 \rho_{\mathrm{i}} \omega-2 \omega \rho_{\mathrm{e}}\left(\frac{k_{\mathrm{i}}}{k_{\mathrm{e}}}\right) Q_{m} \\
& +\rho_{\mathrm{e}}\left(\omega^{2}-\omega_{\mathrm{Ae}}^{2}\right)\left[\frac{\omega^{3}}{v_{\mathrm{si}}^{2}+v_{\mathrm{Ai}}^{2}} \frac{\left(\omega^{2}-2 \omega_{\mathrm{Ci}}^{2}\right)}{\left(\omega^{2}-\omega_{\mathrm{Ci}}^{2}\right)^{2} k_{\mathrm{i}} k_{\mathrm{e}}}\right. \\
& \left.-\frac{\omega^{3}}{v_{\mathrm{se}}^{2}+v_{\mathrm{Ae}}^{2}} \frac{\left(\omega^{2}-2 \omega_{\mathrm{Ce}}^{2}\right) k_{\mathrm{i}}}{\left(\omega^{2}-\omega_{\mathrm{Ce}}^{2}\right)^{2} k_{\mathrm{e}}^{3}}\right] Q_{m} \\
& -\rho_{\mathrm{e}}\left(\omega^{2}-\omega_{\mathrm{Ae}}^{2}\right)\left(\frac{k_{\mathrm{i}}}{k_{\mathrm{e}}}\right)\left[\frac{k_{\mathrm{i}} R P_{m} \omega^{3}\left(\omega^{2}-2 \omega_{\mathrm{Ci}}^{2}\right)}{\left(\omega^{2}-\omega_{\mathrm{si}}^{2}\right)\left(\omega^{2}-\omega_{\mathrm{Ai}}^{2}\right)\left(\omega^{2}-\omega_{\mathrm{Ci}}^{2}\right)}\right. \\
& \left.+\frac{k_{\mathrm{e}} R S_{m} \omega^{3}\left(\omega^{2}-2 \omega_{\mathrm{Ce}}^{2}\right)}{\left(\omega^{2}-\omega_{\mathrm{se}}^{2}\right)\left(\omega^{2}-\omega_{\mathrm{Ae}}^{2}\right)\left(\omega^{2}-\omega_{\mathrm{Ce}}^{2}\right)}\right] \\
& =2 \rho_{\mathrm{i}} \omega-2 \omega \rho_{\mathrm{e}}\left(\frac{k_{\mathrm{i}}}{k_{\mathrm{e}}}\right) Q_{m} \\
& -\rho_{\mathrm{e}} \omega^{3}\left(\omega^{2}-\omega_{\mathrm{Ae}}^{2}\right)\left(\frac{k_{\mathrm{i}}}{k_{\mathrm{e}}}\right)\left[\frac{\omega^{2}-2 \omega_{\mathrm{Ci}}^{2}}{\left(\omega^{2}-\omega_{\mathrm{Si}}^{2}\right)\left(\omega^{2}-\omega_{\mathrm{Ai}}^{2}\right)\left(\omega^{2}-\omega_{\mathrm{Ci}}^{2}\right)}\right. \\
& \left.-\frac{\omega^{2}-2 \omega_{\mathrm{Ce}}^{2}}{\left(\omega^{2}-\omega_{\mathrm{se}}^{2}\right)\left(\omega^{2}-\omega_{\mathrm{Ae}}^{2}\right)\left(\omega^{2}-\omega_{\mathrm{Ce}}^{2}\right)}\right] Q_{m} \\
& -\rho_{\mathrm{e}} \omega^{3}\left(\omega^{2}-\omega_{\mathrm{Ae}}^{2}\right)\left(\frac{k_{\mathrm{i}}}{k_{\mathrm{e}}}\right)\left[\frac{k_{\mathrm{i}} R P_{m}\left(\omega^{2}-2 \omega_{\mathrm{Ci}}^{2}\right)}{\left(\omega^{2}-\omega_{\mathrm{si}}^{2}\right)\left(\omega^{2}-\omega_{\mathrm{Ai}}^{2}\right)\left(\omega^{2}-\omega_{\mathrm{Ci}}^{2}\right)}\right. \\
& \left.+\frac{k_{\mathrm{e}} R S_{m}\left(\omega^{2}-2 \omega_{\mathrm{Ce}}^{2}\right)}{\left(\omega^{2}-\omega_{\mathrm{se}}^{2}\right)\left(\omega^{2}-\omega_{\mathrm{Ae}}^{2}\right)\left(\omega^{2}-\omega_{\mathrm{Ce}}^{2}\right)}\right] \\
& =2 \rho_{\mathrm{i}} \omega-2 \omega \rho_{\mathrm{e}}\left(\frac{k_{\mathrm{i}}}{k_{\mathrm{e}}}\right) Q_{m} \\
& -\rho_{\mathrm{e}} \omega^{3}\left(\omega^{2}-\omega_{\mathrm{Ae}}^{2}\right)\left(\frac{k_{\mathrm{i}}}{k_{\mathrm{e}}}\right) \frac{\left(\omega^{2}-2 \omega_{\mathrm{Ci}}^{2}\right)\left[Q_{m}+k_{\mathrm{i}} R P_{m}\right]}{\left(\omega^{2}-\omega_{\mathrm{si}}^{2}\right)\left(\omega^{2}-\omega_{\mathrm{Ai}}^{2}\right)\left(\omega^{2}-\omega_{\mathrm{Ci}}^{2}\right)} \\
& +\rho_{\mathrm{e}} \omega^{3}\left(\omega^{2}-\omega_{\mathrm{Ae}}^{2}\right)\left(\frac{k_{\mathrm{i}}}{k_{\mathrm{e}}}\right) \frac{\left(\omega^{2}-2 \omega_{\mathrm{Ce}}^{2}\right)\left[Q_{m}-k_{\mathrm{e}} R S_{m}\right]}{\left(\omega^{2}-\omega_{\mathrm{se}}^{2}\right)\left(\omega^{2}-\omega_{\mathrm{Ae}}^{2}\right)\left(\omega^{2}-\omega_{\mathrm{Ce}}^{2}\right)} .
\end{aligned}
$$

Then the imaginary part $\gamma_{m}$ for the surface wave in the slow (cusp) continuum is

$$
\begin{aligned}
\gamma_{m} & =-\frac{D_{m i}\left(\omega=\omega_{r}\right)}{\left.\frac{\partial D_{m r}}{\partial \omega}\right|_{\omega=\omega_{r}}} \\
& =-\frac{\frac{\pi \rho_{\mathrm{e}} k_{z}^{2}}{k_{\mathrm{e}} \rho_{c} \Delta_{c} \mid}\left(\frac{v_{\mathrm{sc}}^{2}}{v_{\mathrm{sc}}^{2}+v_{\mathrm{Ac}}^{2}}\right)^{2}\left(\omega_{r}^{2}-\omega_{\mathrm{Ai}}^{2}\right)\left(\omega_{r}^{2}-\omega_{\mathrm{Ae}}^{2}\right) G_{m}}{2 \omega_{r}\left[1-\chi\left(\frac{k_{\mathrm{i}}}{k_{\mathrm{e}}}\right) Q_{m}\right]-\omega_{r} \chi\left(\frac{k_{\mathrm{i}}}{k_{\mathrm{e}}}\right) T_{m}},
\end{aligned}
$$

where

$$
\begin{aligned}
T_{m}= & \omega_{r}^{2}\left(\omega_{r}^{2}-\omega_{\mathrm{Ae}}^{2}\right)\left\{\frac{\left(\omega_{r}^{2}-2 \omega_{\mathrm{Ci}}^{2}\right)\left[Q_{m}+k_{\mathrm{i}} R P_{m}\right]}{\left(\omega_{r}^{2}-\omega_{\mathrm{si}}^{2}\right)\left(\omega_{r}^{2}-\omega_{\mathrm{Ai}}^{2}\right)\left(\omega_{r}^{2}-\omega_{\mathrm{Ci}}^{2}\right)}\right. \\
& \left.-\frac{\left(\omega_{r}^{2}-2 \omega_{\mathrm{Ce}}^{2}\right)\left[Q_{m}-k_{\mathrm{e}} R S_{m}\right]}{\left(\omega_{r}^{2}-\omega_{\mathrm{se}}^{2}\right)\left(\omega_{r}^{2}-\omega_{\mathrm{Ae}}^{2}\right)\left(\omega_{r}^{2}-\omega_{\mathrm{Ce}}^{2}\right)}\right\} .
\end{aligned}
$$


In the incompressible limit $\left(v_{\mathrm{si}}\left(v_{\mathrm{se}}\right) \rightarrow \infty\right), \gamma_{m}$ reduces to

$\gamma_{m}=-\frac{\frac{\pi \rho_{\mathrm{e}} k_{z}^{2}}{k_{\mathrm{e}} \Delta_{c} \Delta_{c}}\left(\omega_{r}^{2}-\omega_{\mathrm{Ai}}^{2}\right)\left(\omega_{r}^{2}-\omega_{\mathrm{Ae}}^{2}\right) G_{m}}{2 \omega_{r}\left[1-\chi Q_{m}\right]}$,

since $T_{m}$ goes to zero. The damping rate is given as $\gamma_{m} / \omega_{r}$.

\section{Appendix C: Damping rate in long wavelength limit under photospheric conditions}

In the long wavelength limit $\left(\omega_{r} \approx \omega_{\mathrm{Ci}}\right)$, by using Eqs. (15)-(18), we obtain for $T_{0}$ (Eq. (41))

$$
\begin{aligned}
T_{0}= & -\frac{3}{16} \frac{\omega_{\mathrm{Ci}}^{4}\left(\omega_{\mathrm{Ci}}^{2}-\omega_{\mathrm{Ae}}^{2}\right) k_{\mathrm{i}}^{4} R^{4} \ln \left(k_{z} R\right)}{\alpha\left(\omega_{\mathrm{Ci}}^{2}-\omega_{\mathrm{si}}^{2}\right)\left(\omega_{\mathrm{Ci}}^{2}-\omega_{\mathrm{Ai}}^{2}\right)} \\
& +\frac{\omega_{\mathrm{Ci}}^{2}\left(\omega_{\mathrm{Ci}}^{2}-2 \omega_{\mathrm{Ce}}^{2}\right) k_{\mathrm{i}}^{2} R^{2}}{2\left(\omega_{\mathrm{Ci}}^{2}-\omega_{\mathrm{se}}^{2}\right)\left(\omega_{\mathrm{Ci}}^{2}-\omega_{\mathrm{Ce}}^{2}\right)} \\
= & \frac{3}{8} \frac{\omega_{\mathrm{Ci}}^{4}\left(\omega_{\mathrm{si}}^{2}+\omega_{\mathrm{Ai}}^{2}\right) k_{\mathrm{i}}^{4} R^{2}}{\chi\left(\omega_{\mathrm{Ci}}^{2}-\omega_{\mathrm{si}}^{2}\right)^{2}\left(\omega_{\mathrm{Ci}}^{2}-\omega_{\mathrm{Ai}}^{2}\right) k_{z}^{2}} \\
& +\frac{\omega_{\mathrm{Ci}}^{2}\left(\omega_{\mathrm{Ci}}^{2}-2 \omega_{\mathrm{Ce}}^{2}\right) k_{\mathrm{i}}^{2} R^{2}}{2\left(\omega_{\mathrm{Ci}}^{2}-\omega_{\mathrm{se}}^{2}\right)\left(\omega_{\mathrm{Ci}}^{2}-\omega_{\mathrm{Ce}}^{2}\right)} \\
= & \frac{3}{2} \frac{\omega_{\mathrm{Ci}}^{4}\left(\omega_{\mathrm{Ci}}^{2}-\omega_{\mathrm{Ai}}^{2}\right)\left(\omega_{\mathrm{si}}^{2}+\omega_{\mathrm{Ai}}^{2}\right)}{\chi^{3}\left(\omega_{\mathrm{Ci}}^{2}-\omega_{\mathrm{si}}^{2}\right)^{2}\left(\omega_{\mathrm{Ci}}^{2}-\omega_{\mathrm{Ae}}^{2}\right)^{2} k_{z}^{2} R^{2} \ln \left(k_{z} R\right)} \\
& +\frac{\omega_{\mathrm{Ci}}^{2}\left(\omega_{\mathrm{Ci}}^{2}-2 \omega_{\mathrm{Ce}}^{2}\right)\left(\omega_{\mathrm{Ci}}^{2}-\omega_{\mathrm{Ai}}^{2}\right)}{\chi\left(\omega_{\mathrm{Ci}}^{2}-\omega_{\mathrm{se}}^{2}\right)\left(\omega_{\mathrm{Ci}}^{2}-\omega_{\mathrm{Ce}}^{2}\right)\left(\omega_{\mathrm{Ci}}^{2}-\omega_{\mathrm{Ae}}^{2}\right) \ln \left(k_{z} R\right)} \\
= & -\frac{3}{2} \frac{\omega_{\mathrm{Ai}}^{8}}{\chi^{3} \omega_{\mathrm{si}}^{4}\left(\omega_{\mathrm{Ci}}^{2}-\omega_{\mathrm{Ae}}^{2}\right)^{2} k_{z}^{2} R^{2} \ln ^{2}\left(k_{z} R\right)} \\
& -\frac{\omega_{\mathrm{Ci}}^{4} \omega_{\mathrm{Ai}}^{2}\left(\omega_{\mathrm{Ci}}^{2}-2 \omega_{\mathrm{Ce}}^{2}\right)}{\chi \omega_{\mathrm{si}}^{2}\left(\omega_{\mathrm{Ci}}^{2}-\omega_{\mathrm{se}}^{2}\right)\left(\omega_{\mathrm{Ci}}^{2}-\omega_{\mathrm{Ce}}^{2}\right)\left(\omega_{\mathrm{Ci}}^{2}-\omega_{\mathrm{Ae}}^{2}\right) \ln \left(k_{z} R\right)}
\end{aligned}
$$

Under photospheric conditions $\left(\omega_{\mathrm{Ce}}\left(\omega_{\mathrm{Ae}}\right) \approx 0\right)$, Eq. $(40)$ then becomes

$$
\begin{aligned}
\gamma_{0} & =-\frac{\frac{\pi \chi k_{z}^{2} R}{\left|\Delta_{c}\right|}\left(\frac{v_{\mathrm{si}}^{2}}{v_{\mathrm{si}}^{2}+v_{\mathrm{Ai}}^{2}}\right)^{2}\left(\omega_{\mathrm{Ci}}^{2}-\omega_{\mathrm{Ai}}^{2}\right)\left(\omega_{\mathrm{Ci}}^{2}-\omega_{\mathrm{Ae}}^{2}\right) \ln \left(k_{z} R\right)}{2 \omega_{\mathrm{Ci}}\left(\frac{\omega_{\mathrm{Ai}}^{2}}{\omega_{\mathrm{Ci}}^{2}}\right)-\chi \omega_{\mathrm{Ci}} T_{0}} \\
& =-\frac{\pi \chi k_{z}^{2} R l\left(\frac{\omega_{\mathrm{Ci}}^{2}}{\omega_{\mathrm{Ai}}^{2}}\right)^{2}\left(\omega_{\mathrm{Ci}}^{2}-\omega_{\mathrm{Ai}}^{2}\right) \ln \left(k_{z} R\right)}{4 \omega_{\mathrm{Ci}}\left(\frac{\omega_{\mathrm{Ai}}^{2}}{\omega_{\mathrm{Ci}}^{2}}\right)-2 \chi \omega_{\mathrm{Ci}} T_{0}},
\end{aligned}
$$

where $T_{0}$ is given as

$$
\begin{aligned}
T_{0}= & -\frac{3}{2} \frac{\omega_{\mathrm{Ai}}^{8}}{\chi^{3} \omega_{\mathrm{si}}^{4}\left(\omega_{\mathrm{Ci}}^{2}-\omega_{\mathrm{Ae}}^{2}\right)^{2} k_{z}^{2} R^{2} \ln ^{2}\left(k_{z} R\right)} \\
& -\frac{\omega_{\mathrm{Ci}}^{4} \omega_{\mathrm{Ai}}^{2}\left(\omega_{\mathrm{Ci}}^{2}-2 \omega_{\mathrm{Ce}}^{2}\right)}{\chi \omega_{\mathrm{si}}^{2}\left(\omega_{\mathrm{Ci}}^{2}-\omega_{\mathrm{se}}^{2}\right)\left(\omega_{\mathrm{Ci}}^{2}-\omega_{\mathrm{Ce}}^{2}\right)\left(\omega_{\mathrm{Ci}}^{2}-\omega_{\mathrm{Ae}}^{2}\right) \ln \left(k_{z} R\right)} \\
= & -\frac{3}{2} \frac{\omega_{\mathrm{Ai}}^{8}}{\chi^{3} \omega_{\mathrm{Ci}}^{4} \omega_{\mathrm{si}}^{4} k_{z}^{2} R^{2} \ln \left(k_{z} R\right)^{2}}-\frac{\omega_{\mathrm{Ci}}^{2} \omega_{\mathrm{Ai}}^{2}}{\chi \omega_{\mathrm{si}}^{2}\left(\omega_{\mathrm{Ci}}^{2}-\omega_{\mathrm{se}}^{2}\right) \ln \left(k_{z} R\right)} .
\end{aligned}
$$

Thus we can have the expression for the damping rate $\gamma_{0} / \omega_{\mathrm{Ci}}$ as

$$
\begin{aligned}
\frac{\gamma_{0}}{\omega_{\mathrm{Ci}}}=-\frac{\pi \chi k_{z}^{2} R l\left(\frac{\omega_{\mathrm{Ci}}^{2}}{\omega_{\mathrm{Ai}}^{4}}\right)\left(\omega_{\mathrm{Ci}}^{2}-\omega_{\mathrm{Ai}}^{2}\right) \ln \left(k_{z} R\right)}{4\left(\frac{\omega_{\mathrm{Ai}}^{2}}{\omega_{\mathrm{Ci}}^{2}}\right)+\left[\frac{3 \omega_{\mathrm{Ai}}^{8}}{\chi^{2} \omega_{\mathrm{Ci}}^{4} \omega_{\mathrm{si}}^{4} k_{z}^{2} R^{2} \ln ^{2}\left(k_{z} R\right)}+\frac{2 \omega_{\mathrm{Ci}}^{2} \omega_{\mathrm{Ai}}^{2}}{\omega_{\mathrm{si}}^{2}\left(\omega_{\mathrm{Ci}}^{2}-\omega_{\mathrm{se}}^{2}\right) \ln \left(k_{z} R\right)}\right]} \\
=\frac{\pi \chi(l / R)\left(\frac{\omega_{\mathrm{Ci}}^{4}}{\omega_{\mathrm{si}}^{2} \omega_{\mathrm{Ai}}^{2}}\right) k_{z}^{2} R^{2} \ln \left(k_{z} R\right)}{\frac{4 \omega_{\mathrm{Ai}}^{2}}{\omega_{\mathrm{Ci}}^{2}}+\left[\frac{3 \omega_{\mathrm{Ai}}^{8}}{\chi^{2} \omega_{\mathrm{Ci}}^{4} \omega_{\mathrm{si}}^{4} 2_{z}^{2} R^{2} \ln ^{2}\left(k_{z} R\right)}+\frac{2 \omega_{\mathrm{Ci}}^{2} \omega_{\mathrm{Ai}}^{2}}{\omega_{\mathrm{si}}^{2}\left(\omega_{\mathrm{Ci}}^{2}-\omega_{\mathrm{se}}^{2}\right) \ln \left(k_{z} R\right)}\right]} .
\end{aligned}
$$

